\title{
Motivational salience guides attention to valuable and threatening stimuli: Evidence from behaviour and fMRI
}

Haena Kim, Namrata Nanavaty, Humza Ahmed, Vani A. Mathur \& Brian A. Anderson

Texas A\&M University

To whom correspondence should be addressed:

Haena Kim

Texas A\&M University

Department of Psychological and Brain Sciences

4235 TAMU

College Station, TX 77843-4235

E-mail: hannah.kim@tamu.edu 


\title{
MOTIVATIONAL SALIENCE GUIDES ATTENTION
}

\begin{abstract}
Rewarding and aversive outcomes have opposing effects on behaviour, facilitating approach and avoidance, although we need to accurately anticipate each type of outcome in order to behave effectively. Attention is biased toward stimuli that have been learned to predict either type of outcome, and it remains an open question whether such orienting is driven by separate systems for value- and threat-based orienting or whether there exists a common underlying mechanism of attentional control driven by motivational salience. Here we provide a direct comparison of the neural correlates of value- and threat-based attentional capture following associative learning. Across multiple measures of behaviour and brain activation, our findings overwhelmingly support a motivational salience account of the control of attention. We conclude that there exists a core mechanism of experience-dependent attentional control driven by motivational salience, and that prior characterisations of attention as being value-driven or supporting threat monitoring need to be revisited.
\end{abstract}

\section{Keywords}

selection history, aversive conditioning, motivational salience, fMRI, eye tracking 


\section{MOTIVATIONAL SALIENCE GUIDES ATTENTION}

\section{Introduction}

Attention selectively processes perceptual information, helping to ensure that stimuli relevant to survival and well-being are preferentially represented by the brain (Corbetta \& Shulman, 2002; Desimone \& Duncan, 1995). Traditionally, the allocation of limited attentional resources had been thought to be governed by task goals (Wolfe, Cave, \& Franzel, 1989) and physical salience (Theeuwes, 2010). A newer construct, selection history, challenges this dichotomy and suggests previous episodes of attentional orienting are capable of independently biasing attention in a manner that is neither top-down nor bottom-up (Awh, Belopolsky, \& Theeuwes, 2012). One component of selection history is reward history. Via associative learning, initially neutral stimuli come to predict reward and thus acquire heightened attentional priority, consequently capturing attention even when non-salient and task-irrelevant (referred to as valuedriven attentional capture; e.g., Anderson, Laurent, \& Yantis, 2011).

The dopamine system is implicated in value-driven attentional capture. Increased dopamine release in the basal ganglia leads to stronger attentional bias by stimuli with reward history (Anderson et al., 2016; Anderson et al., 2017) and in particular, the caudate tail responds preferentially to such stimuli (Anderson, Laurent, \& Yantis, 2014; Yamamoto, Kim, \& Hikosaka, 2013). These findings corroborate the literature on the role of dopamine in formulating reward behaviour; prediction error signals facilitate outcome-maximising decisions (O'Doherty, 2004; Schultz, Dayan, \& Montague, 1997). Through repetition, the caudate tail comes to encode stable value information (H. F. Kim \& Hikosaka, 2013), which eventually contributes to incentive salience in which the reward-predictive stimuli automatically elicit an approach bias (Berridge \& Robinson, 1998).

The influence of prior experience shaped by aversive outcomes on the allocation of attention is beginning to be explored. Behaviourally, aversive outcomes bias attention in a similar manner even when non-salient and task-irrelevant (Nissens, Failing, \& Theeuwes, 2017; Schmidt, Belopolsky, \& Theeuwes, 2015a, 2015b; Wentura, Müller, \& Rothermund, 2014), suggesting that the attentional system is primarily guided by motivational salience rather than a particular emotional valence. According to the motivational relevance model, both reward and aversive outcomes are important for survival (Gable \& Harmon-Jones, 2010), hence eliciting automatic attentional orienting that facilitates approach-avoidance behaviour (LeDoux, 1996; Vuilleumier, 2005).

Less is known about the neural mechanisms of attentional bias following aversive conditioning and whether there exists a similar neural profile between value- and threat-based orienting. Brain regions such as the striatum, ventral tegmental area and substantia nigra process not only reward but also aversive outcomes (Becerra, Breiter, Wise, Gonzalez, \& Borsook, 2001; J. Jensen et al., 2003; Liu, Hairston, Schrier, \& Fan, 2011). A subpopulation of dopamine neurons excites to both reward and aversive outcomes (Bromberg-Martin, Matsumoto, \& Hikosaka, 2010; Horvitz, 2000), suggesting aversive conditioning may bias attention in a manner similar to valuedriven attention, possibly via the nigrostriatal pathway that controls oculomotor movement (Hikosaka, Nakamura, \& Nakahara, 2006; Hikosaka, Takikawa, \& Kawagoe, 2000). Such findings are consistent with the hypothesis that the attentional system is primarily guided by motivational salience. However, such regional overlap does not necessitate a similar neural profile with respect to the control of attention. Indeed, reward and aversive outcomes are also represented in dissociable neural systems (Baliki, Geha, Fields, \& Apkarian, 2010; Yacubian et al., 2006). Alternatively, the two outcomes may be represented along a bipolar continuum; the same regions are excited following reward and suppressed following an aversive outcome (Becerra \& Borsook, 2008; Delgado, Nystrom, Fissell, Noll, \& Fiez, 2000), consistent with the traditional view that dopamine neurons encode value signals (Schultz et al., 1997). This differential encoding has consequences for action selection, in that reward promotes approach and aversive outcomes promote inhibition or avoidance (Chen \& Bargh, 1999; O'Doherty, 2004). Such dissociable outcome representations could also have dissociable influences on the attention system, suggesting at least two separate mechanisms by which motivationally relevant stimuli capture attention.

Here, we present two experiments that examined the neural correlates of attentional bias following aversive conditioning (Experiment 1) and the influence of reward and aversive 


\section{MOTIVATIONAL SALIENCE GUIDES ATTENTION}

outcomes on attentional bias (Experiment 2) using functional magnetic resonance imaging (fMRI). In Experiment 1, participants completed a training phase in which each of two differently coloured circles was either followed by a mildly painful heat pulse applied to their left forearm (CS+) or never paired with a heat pulse (CS-). A subsequent test phase involved searching for a shapedefined target among non-salient distractors. Sometimes, one of the distractors appeared in either the CS+ or CS- colour (see Figure 1). In Experiment 2, in a training phase, participants learned to associate colours with either a reward (monetary gain), threat (unavoidable electric shock), or no outcome (neutral). In a test phase, a distractor square and a target circle were presented simultaneously, one of which could appear in either the previously reward- or threatassociated colour (see Figure 2). Experiment 1 provided an opportunity to characterise the neural correlates of automatic attentional processing of aversively-conditioned stimuli. We found that such attentional processing recruits brain regions which are also implicated in value-driven attentional capture with substantial apparent overlap, suggesting that attentional bias towards reward and aversive outcomes involve a common underlying mechanism. Motivated by these findings, Experiment 2 afforded a direct comparison between such neural correlates and the neural correlates of value-driven attention. If there exist genuinely dissociable neural correlates between attentional bias towards reward and aversive outcomes, then we would expect to find a unique pattern of activation in response to distractors that signal reward and aversive outcomes.

\section{Materials and Methods Experiment 1 Participants}

Thirty healthy participants ( 15 females; mean age $=22.4$ years) were recruited from the Texas A\&M University community. All participants had normal or corrected-to-normal visual acuity, normal colour vision, no recent history of chronic pain, no current acute pain or injury, and had not taken any pain medication for at least three days prior to the study. All procedures were approved by the Texas A\&M University Institutional Review Board and conformed with the principles outlined in the Declaration of Helsinki.

\section{Apparatus}

For the in-lab portion of the experiment, stimulus presentation was controlled by a standard Windows desktop equipped with MATLAB and Psychtoolbox 3.0. The eye-to-screen distance was approximately $70 \mathrm{~cm}$. For the $\mathrm{fMRI}$ portion of the experiment, stimulus presentation was controlled by an Invivo SensaVue display system. Key responses were entered using a Cedrus Lumina two-button response pad. The eye-to-screen distance was approximately $125 \mathrm{~cm}$. The heat stimulus for in-lab and fMRI procedures was delivered to the left volar forearm with a contact probe (30×30 mm Medoc Pathway ATS Peltier device; Medoc Advanced Medical Systems Ltd., Ramat Yishai, Israel).

\section{Procedure}

The study required a lab visit and an fMRI scan visit on the following day. During the lab visit, participants completed a quantitative thermal testing protocol and temperature calibration procedure (to equate perceptual intensity of the aversive stimulus and control for individual differences in pain sensitivity), followed by two runs of the training phase and a practice run for the test phase. During the scan visit, participants repeated the calibration procedure and then completed nine brain scans, including two training runs, followed by three test runs, an anatomical scan, another training run and two test runs.

Thermal testing protocol and temperature calibration. Participants completed three quantitative sensory testing procedures during the laboratory visit to determine individualised range of thermal pain thresholds and tolerances, map changes in individual pain intensity with increasing temperature levels, and test consistency of evoked pain across stimulus temperatures (Mathur et al., 2016). All three procedures were considered to ensure that the training stimulus was perceived as painful (suprathreshold yet tolerable), and was the temperature that evoked (or most closely evoked) a "7" on a 0 (no pain) to 10 (worst pain imaginable) numerical pain rating 


\section{MOTIVATIONAL SALIENCE GUIDES ATTENTION}

scale. During the fMRI visit, stimulus temperature was first confirmed or updated in the scan environment.

Training phase. Each training run consisted of 30 trials. Each trial began with a fixation cross for $1.8 \mathrm{~s}$, followed by a CS display for $3 \mathrm{~s}$ and a blank screen for $1.8-5.4 \mathrm{~s}$. The CS display contained either a red or a green circle $\left(4.3^{\circ}\right.$ in diameter) in the centre of the screen, one of which was probabilistically followed by a heat pulse (CS+). The other one was never followed by a heat pulse (CS-). The CS-colour mapping was counterbalanced across participants. In each run, half of the trials were CS+ trials and the other half were CS- trials. Two-thirds of the CS+ trials were followed by a heat pulse. The remaining one-third of the CS+ trials and all CS- trials were not followed by a heat pulse. On CS+ trials followed by a heat pulse, a heat pulse was delivered $1.2 \mathrm{~s}$ post the CS display onset. Participants were instructed to observe circles presented on the screen and also informed that they would sometimes feel heat (Figure 1).

Test phase. Each test run consisted of 60 trials. Each trial began with a fixation cross for $1.8 \mathrm{~s}$, followed by a search display for $1.8 \mathrm{~s}$ and a blank screen for 0.6-4.2 $\mathrm{s}$. The search display consisted of six uniquely coloured shapes $\left(2.7^{\circ} \times 2.7^{\circ}\right)$. One of the shapes was a shape singleton target and the rest were differently shaped distractors. On each side of the display, the middle shape was presented $9.1^{\circ}$ from the fixation cross and the top and bottom shapes were presented $8.5^{\circ}$ from the fixation cross. On one-third of the 60 trials, one of the distractors appeared in the CS+ colour and on another one-third, it appeared in the CS- colour. The remaining one-third were CS distractor absent trials (did not contain either colour presented during training). For each distractor condition, the target was presented on each side of the screen equally-often, and for the CS+ and CS- distractor conditions, the distractor position was pseudo-randomised such that it was presented on the opposite side of the screen as the target on $3 / 5$ of trials and on the same side on $2 / 5$ of trials (corresponding to the distribution of the five remaining non-target positions), separately for targets on the left and right. On half of the 60 trials, the target was a circle and the distractors were diamonds and on the other half, the mapping was reversed. All shapes had a line segment in it. Inside the target, it was tilted either horizontally or vertically and inside the nontargets, it was tilted $45^{\circ}$ either to the left or to the right. Participants reported the orientation of a line within the target by pressing the left button for a vertical line and the right button for a horizontal line on the response pad using their right hand. Practice for the test phase consisted of 30 CS distractor absent trials (Figure 1).

\section{MRI data acquisition}

MRI data were acquired with a Siemens 3-Tesla MAGNETOM Verio scanner and a 32channel head coil at the Texas A\&M Institute for Preclinical Studies (TIPS). An anatomical image was acquired using a magnetisation prepared rapid gradient echo (MPRAGE) T1-weighted sequence $\left(150\right.$ coronal slices, $\mathrm{TR}=7.9 \mathrm{~ms}, \mathrm{TE}=3.65 \mathrm{~ms}$, flip angle $=8^{\circ}$, voxel size $=1 \mathrm{~mm}$ isotropic). Whole-brain functional T2*-weighted images were acquired using a multiband echo planar imaging (EPI) sequence (multiband factor $=8,56$ axial slices, $\mathrm{TR}=600 \mathrm{~ms}, \mathrm{TE}=29 \mathrm{~ms}$, flip angle $=52^{\circ}$, image matrix $=96 \times 96$, field of view $=240 \mathrm{~mm}$, slice thickness $=2.5 \mathrm{~mm}$ with no gap). All functional scans began with dummy pulses to allow stabilisation of magnetic fields.

\section{MRI data processing}

Data from one participant were discarded prior to data analysis due to below chance performance in the test phase. MRI data were preprocessed and analysed using the AFNI software package. All functional images were first motion corrected, co-registered to the anatomical image of each participant and warped to the Talairach brain using 3dQwarp. The images were then normalised to the mean signal intensity of each run and spatially smoothed to a resulting $5 \mathrm{~mm}$ full width half maximum Gaussian kernel using 3dBlurToFWHM. The preprocessed images from the training phase were fitted to a general linear model (GLM) with the following regressors: (1) CS+ circle followed by a heat pulse (2) CS+ circle not followed by a heat pulse and (3) CS- circle. For the images from the test phase, we used the following: (1) target on the left, distractor absent, (2) target on the right, distractor absent, (3) target on the left, CS+ 


\section{MOTIVATIONAL SALIENCE GUIDES ATTENTION}

distractor on the right, (4) target on the right, CS+ distractor on the left, (5) target on the left, CSdistractor on the right, (6) target on the right, CS- distractor on the left. Regressors of non-interest included trials on which the CS distractor and target were presented on the same side of the screen (separately for each combination, as in Anderson et al., 2014), six motion parameters and scanner drift. The regressors were modelled using a finite impulse response (FIR) function beginning at the onset of the CS display and search display for the training and test phase, respectively. We then extracted the maximum beta-weights from a time window of 3-6 seconds post search display onset, reflecting the peak of the stimulus-evoked response.

\section{MRI data analysis}

Training data. We performed two paired-samples $t$-tests, one comparing CS+trials followed by a heat pulse versus CS- trials, and one comparing CS+ trials not followed by a heat pulse versus CS- trials. The contrast images were thresholded at voxelwise $p<0.01$ and corrected for multiple comparisons using the AFNI programme 3dClustSim, with the smoothness of the data estimated using the ACF method (clusterwise $\alpha<0.05$, cluster size $k \geq 24$ ).

Test data. Given our a priori hypotheses informed by results we previously reported using a similar paradigm, four paired-samples $t$-tests were performed, each comparing trials on which either the CS+ or CS- distractor was presented in the contralateral hemifield and those without the CS distractor but the target was presented in the ipsilateral hemifield in each case (thus more effectively isolating the representation of task-irrelevant stimuli as a function of selection history, see Anderson et al., 2014; Haena Kim \& Anderson, 2019b). The resulting contrast images were thresholded in the same way as the training data. For the bilateral amygdala and substantia nigra regions of interest (ROI) analyses, we extracted beta coefficients from each anatomically-defined $\mathrm{ROI}$ and performed the same four paired-samples $t$-tests.

\section{Behavioural data analysis}

Data from two participants were excluded due to equipment failure. Correct response times (RTs) from the test runs faster than 200 ms or exceeding 2.5 standard deviations of the conditional mean were trimmed. RT and accuracy data were initially subjected to a $3 \times 5$ ANOVA, with distractor condition (CS+ distractor, CS- distractor, CS distractor absent) and run (1-5) as factors. There was no significant effect on accuracy (all $p s>0.41$ ) other than a main effect of run, $F(4,104)=7.99, p<0.001, \eta_{p}^{2}=0.24$. Following a significant interaction effect on $\mathrm{RT}, F(8,208)$ $=3.88, p<0.001, \eta_{p}^{2}=0.13$, we compared the distractor conditions by performing a $2 \times 5$ ANOVA for each possible distractor condition pair (assessing partial interactions). Comparing the CS+ distractor to (1) CS- distractor and (2) CS distractor absent condition revealed a significant interaction effect in each case, $p s<0.01$. However, there was no significant interaction effect for the CS- distractor and CS distractor absent condition pair, $F(4,104)=1.18, p=0.322$, suggesting no quantitative difference between the two conditions. Based on these results, we collapsed the CS- distractor and CS distractor absent conditions in all subsequent analyses.

It is possible that by the time the third test run begins, the effect of the first two training runs dissipates due to extinction and the CS+ distractor no longer captures attention. We therefore grouped the test runs based on their temporal proximity to the most recent training run to capture any effect of extinction (which is generally more informative given the interleaved training phase - test phase design). Specifically, we categorised the test runs into three groups based on their temporal relationship to the most recent training run. Test runs that immediately followed a training run were labelled post training 1 , those that followed second were post training 2 and the last run was post training 3. RT data were therefore subjected to a $2 \times 3$ ANOVA, with distractor condition (CS+ distractor, non-CS+ distractor) and post training run (1-3) as factors. Following a significant interaction, we performed paired-samples $t$-tests for planned contrasts. When appropriate, we report Greenhouse-Geisser corrected $p$-values.

\section{Experiment 2 \\ Participants}




\title{
MOTIVATIONAL SALIENCE GUIDES ATTENTION
}

Forty-two healthy participants ( 16 females; mean age $=21.6$ years) were recruited from the Texas A\&M University community. All participants had normal or corrected-to-normal visual acuity and normal colour vision. All procedures were approved by the Texas A\&M University Institutional Review Board and conformed with the principles outlined in the Declaration of Helsinki.

\begin{abstract}
Apparatus
For the in-lab portion of the experiment, stimulus presentation was controlled by a standard Windows desktop equipped with MATLAB and Psychtoolbox 3.0. The eye-to-screen distance was approximately $70 \mathrm{~cm}$. Eye position was monitored using an EyeLink 1000 Plus desktop mount eye tracker. Electric shocks were generated by an isolate linear stimulator (BIOPAC) operating in current mode. For the fMRI portion of the experiment, the general set-up was similar to that of Experiment 1, except that eye position was monitored using an EyeLink 1000 Plus tower mount eye tracker and electric shocks were generated using a BIOPAC MP160 system.
\end{abstract}

\section{Procedure}

The study required a lab visit and a scan visit on the following day. During the lab visit, participants completed a shock calibration procedure, practice run for the training phase, six runs of the training phase and a practice run for the test phase. The shock intensity was individually adjusted during the calibration procedure by gradually increasing it to a level where participants perceived it as uncomfortable but not painful (as in, e.g., Haena Kim \& Anderson, in press; Nissens et al., 2017; Schmidt et al., 2015b). Each task run began with five-point eye position calibration. During the scan visit, participants repeated the shock calibration procedure and then completed ten brain scans, including two training runs, followed by three test runs, an anatomical scan, another training run and three test runs.

Training phase. Each training run consisted of 40 trials. Each trial began with a fixation display for $1.8 \mathrm{~s}$, followed by a stimulus display for $0.6 \mathrm{~s}$, a blank screen for $1.2 \mathrm{~s}$, a feedback display for $1.8 \mathrm{~s}$ and a fixation display for $0.6-4.2 \mathrm{~s}$. The fixation display contained a fixation cross at the centre. The stimulus display had a square $\left(3.7^{\circ} \times 3.7^{\circ}\right)$, presented $9.2^{\circ}$ either on the left or right of the fixation cross, to which participants had to generate a saccade. Correct saccades to the target immediately terminated the trial. The square appeared on each side equally often and it was rendered in one of four equiluminant colours (orange, blue, green and grey) equally often (colour and location fully counterbalanced). Two of the colours predicted no outcome (neutral), and the rest predicted either a reward or shock outcome. The colour-outcome mapping was counterbalanced. The feedback display showed the word "Correct" if participants correctly made a saccade to the square and "Incorrect" if they failed to do so. On shock trials, a mild shock was delivered simultaneously with the feedback display. On reward trials, participants received 50 cents ( $\$ 0.50$ USD) for a correct response. Shock and reward outcomes occurred on $80 \%$ of trials. Practice for the training phase consisted of 20 trials; the stimulus display contained a white square and no outcome was delivered (Figure 2).

Test phase. Each test run consisted of 80 trials. Each trial began with a fixation cross for $1.8 \mathrm{~s}$, followed by a search display for $0.8 \mathrm{~s}$ and a blank screen for $1-4 \mathrm{~s}$. The search display consisted of a distractor square $\left(2.7^{\circ} \times 2.7^{\circ}\right)$ and a target circle $\left(2.7^{\circ}\right.$ in diameter $)$, presented equidistant from the fixation cross on the left and right. The target circle appeared on each side equally often. Participants were instructed to generate a saccade to the circle, regardless of its colour. Saccades that remained in the target circle window (twice larger in width, five times larger in height than the target) for more than $100 \mathrm{~ms}$ were scored as correct. If participants made a saccade to the distractor square window (same size as the target window), the trial was scored as containing an errant eye movement. Targets that appeared in either the reward or shock colour were always paired with one of the two neutral colours and targets that appeared in one of the two neutral colours could be paired with either the reward, shock or the other neutral colour, resulting in five target-distractor combinations (reward target-neutral distractor, shock target- 


\section{MOTIVATIONAL SALIENCE GUIDES ATTENTION}

neutral distractor, neutral target-neutral distractor, neutral target-reward distractor and neutral target-shock distractor). Each combination was presented equally often in each run. Practice for the test phase consisted of 20 trials on which the target and distractor shapes appeared in white (Figure 2).

\section{MRI data acquisition}

MRI set up was identical to that of Experiment 1.

\section{MRI data processing}

Data from nine participants were discarded prior to data analysis due to withdrawal prior to study completion $(n=6)$, low performance $(n=1)$, motion artefact $(n=1)$ and equipment failure (inability to track eye position in the scanner environment; $n=1$ ). Functional images from the test runs were included in the analysis. All procedures leading up to fitting a GLM were equivalent to Experiment 1. We performed two GLMs, one for defining ROIs and one for multivariate pattern analysis (MVPA). The first GLM included the following regressors of interest, collapsed across task runs: (1) reward target and neutral distractor, (2) shock target and neutral distractor, (3) reward distractor and neutral target, (4) shock distractor and neutral target and (5) neutral target and neutral distractor. Unlike in Experiment 1, we decided not to model the data separately based on the side of the display on which targets/distractors appeared in order to obtain a more stable measure of the haemodynamic response given the greater number of experiment conditions and resulting fewer trials-per-cell. The second GLM was equivalent to the first GLM, except that it was performed separately for each run for the purposes of pattern analysis on the resulting beta weight maps (as in Anderson, 2017b). As in Experiment 1, the regressors were modelled using an FIR function beginning at the onset of the search display (see Figure 3). Scanner drift and motion parameters were included as regressors of non-interest. We then extracted the maximum beta-weights from a time window of 3-6 seconds post search display onset. We maintained the use of data smoothed to a resulting $5 \mathrm{~mm}$ full width half maximum given that modestly smoothing data for the purposes of MVPA can result in improved classification accuracy presumably by reducing the influence of noise in the signal (Gardumi et al., 2016; Op de Beeck, 2010).

\section{MRI data analysis}

Whole-brain analysis. Three contrast images were created via a paired-samples $t$-test, one that directly compares the reward and shock distractors, one that compares the reward and neutral distractors and one that compares the shock and neutral distractors. The contrast directly comparing reward and shock distractors was assessed for significance using the same approach to cluster correction as the contrasts computed in Experiment 1.

MVPA. Using the leave-one-subject-out approach to preserve independence (Esterman, TamberRosenau, Chiu, \& Yantis, 2010), we identified 11 ROIs commonly activated by the reward and shock distractors in the reward vs. neutral distractors and shock vs. neutral distractors contrast images described above for each participant. Specifically, we created 33 sets of these two contrast images, each with data from 32 participants (i.e., excluding the "left-out" participant); for each set, we combined the two contrast images to identify commonly activated regions and located the $11 \mathrm{ROIs}$, which served as the ROls for the left-out participant. To maximise sensitivity to regions of overlap, we set the threshold for each contrast liberally at $p<0.05$ voxelwise and determined clusters of voxels for which there was overlap (i.e., the intersection of the two contrasts). Within each region, we extracted beta-weights for the reward and shock distractors (computed as described above), separately for each run. The beta-weights were then standardised (z-scored) and subjected to an MVPA using the linear support vector machine classifier (fitscvm) in MATLAB. The classifier was trained to distinguish the reward and shock distractors using the leave-one-run-out approach and tested on the left out run (as in Anderson, 2017b; Xu et al., 2017), resulting in six classification accuracies. These accuracies were averaged to generate the mean classification accuracy per participant, which were then averaged across participants to compute a grand mean. For each participant, this procedure was then 


\section{MOTIVATIONAL SALIENCE GUIDES ATTENTION}

repeated 10,000 times with the labels randomly shuffled on each iteration; the actual grand mean was compared against the distribution of mean accuracies obtained using this randomisation procedure to quantify the probability of our data under the null hypothesis (i.e., assess for statistical significance). The same MVPA approach was also adopted using all of the clusters for which (a) reward distractors evoked stronger activation than neutral distractors and (b) shock distractors evoked stronger activation than neutral distractors as ROls. To verify the sensitivity of the ROls to detect an actual difference in the pattern of activation, we ran a separate GLM in which the side of the target (left vs. right) was modelled (regardless of valence) and the resulting peak beta weights were subjected to the same MVPA analyses using the same ROls. Portions of this research were conducted with high performance research computing resources provided by Texas A\&M University (https://hprc.tamu.edu).

Sensitivity power analysis. In order to further contextualise non-significant classification in the primary MVPA analyses, we conducted a sensitivity power analysis using simulated data. Using the reward > neutral and shock > neutral ROls, we generated random voxel values (beta value for peak response) for each participant, condition, and run from a distribution that mirrored the variability in signal intensity in the actual data (which was matched one-to-one on a participant/condition/run basis). Then, we increased the signal intensity of a subset of voxels in each condition by a bias factor, and then conducted MVPA as in the primary analysis, repeating the procedure 10,000 times across participants to produce a distribution of classification accuracy. In the simulation, one-third of the voxels were biased to respond more strongly to the reward condition, one-third to the shock condition, and the remaining one-third were undifferentiated (no bias factor applied in either condition). The percentage of classification accuracy above the $p<0.05$ threshold from the randomisation test from the primary analysis using the relevant ROI was determined, and the bias factor increased and the procedure repeated until this percentage first exceeded $80 \%$. In this way, we determined the percent increase in signal intensity for each distractor condition necessary to produce a significant result $80 \%$ of the time, separately for each of the two ROIs, under the assumption that voxels favouring reward-associated distractors, voxels favouring shock-associated distractors, and undifferentiated voxels would be evenly distributed in each ROI.

\section{Behavioural data analysis}

Data from the nine participants not included in the MRI analysis were discarded. RTs faster than 70 ms or exceeding 2.5 standard deviations of the conditional mean were trimmed. Error rate was defined as the proportion of trials containing an initial eye movement to the distractor. We performed an ANOVA to compare all combinations of target and distractor colours (reward target-neutral distractor, shock target-neutral distractor, neutral target-neutral distractor, neutral target-reward distractor and neutral target-shock distractor), separately for RT and error rate, and paired-samples $t$-tests for planned contrasts.

\section{Results \\ Behaviour}

Experiment 1. Preliminary analyses on the test phase RTs from Experiment 1 suggested no differences between the non-CS+ distractor conditions (CS- distractor and CS distractor absent), which were collapsed (see Behavioural data analysis for Experiment 1). There was a significant main effect of run, $F(2,54)=20.51, p<0.001, \eta_{p}^{2}=0.43$, no main effect of distractor condition, $F(1,27)=0.42, p=0.52$, and a significant interaction between distractor condition and run, $F(2$, $54)=15.17, p<0.001, \eta_{p}^{2}=0.36$. The CS+ distractor slowed RTs in the run that immediately followed a training run, $t(26)=3.09, p=0.005, d=0.59$, indicative of attentional capture by stimuli previously associated with an aversive outcome (Haena Kim \& Anderson, in press; Nissens et al., 2017; Schmidt et al., 2015a, 2015b). However, the capture effect disappeared in post training run $2, t(26)=0.56, p=0.58$ and the pattern reversed in post training run 3 ; the CS+ 


\section{MOTIVATIONAL SALIENCE GUIDES ATTENTION}

distractor facilitated RT, $t(26)=-4.26, p<0.001, d=0.82$ (Figure 4), potentially reflecting signal suppression (Gaspelin, Leonard, \& Luck, 2015).

Experiment 2. The test phase in Experiment 2 afforded an opportunity to directly compare the effects of reward learning and aversive conditioning on attentional bias. An ANOVA comparing all five trial types (reward target-neutral distractor, shock target-neutral distractor, neutral targetneutral distractor, neutral target-reward distractor and neutral target-shock distractor) revealed significant differences in RT, $F(4,128)=22.76, p<0.001, \eta_{p}^{2}=0.42$ (Figure 5). The reward and shock distractors slowed RTs relative to the neutral distractors, $t(32)=5.08, p<0.001, d=0.89$ and $t(32)=4.72, p<0.001, d=0.82$, respectively. In contrast, the reward and shock targets facilitated RTs relative to the neutral targets, $t(32)=-3.42, p=0.002, d=0.59$ and $t(32)=-3.04, p$ $=0.005, d=0.52$, respectively.

A similar pattern was observed in error rates, $F(4,128)=16.85, p<0.001, \eta_{p}^{2}=0.35$. Participants made more errors when they needed to look away from the reward and shock distractors, $t(32)=4.4, p<0.001, d=0.77$ and $t(32)=3.16, p=0.003, d=0.55$, respectively. However, they made fewer errors when the target was previously associated with reward and shock, $t(32)=-2.90, p=0.007, d=0.5$ and $t(32)=-2.33, p=0.026, d=0.41$, respectively. Importantly, the facilitatory effects of the reward and shock targets on RT and error rate were comparable in magnitude, all $p s>0.26$. The impeding effects of the reward and shock distractors on RT and error rate were also comparable, all $p s>0.18$. These results confirmed that reward-related and aversively-conditioned stimuli share a similar behavioural profile.

\section{Neuroimaging}

Experiment 1. In the training phase of Experiment 1, relative to the CS- circle, the CS+ circle activated the brain regions involved in pain processing, including the bilateral insula, thalamus, secondary somatosensory cortex and caudate head (Brooks, Nurmikko, Bimson, Singh, \& Roberts, 2002; Freund et al., 2009; K. B. Jensen et al., 2016; Navratilova \& Porreca, 2014; Wager et al., 2013; Woo et al., 2017). Importantly, these regions responded to the CS+ circle even when it was not followed by a heat pulse (Figure 6). Such consistent activation to the CS+ circle regardless of heat pulse delivery indicates successful acquisition of the CS-US association.

We then examined the influence of the CS+ and CS- distractors on attention by comparing the test phase trials on which either the CS+ or CS- distractor was present in the contralateral hemifield and those on which the CS distractor was absent while the target was present in the ipsilateral hemifield in each case. The CS+ distractor activated the brain regions within the frontoparietal attention network. When it was present in the left hemifield, significant activations were observed in the bilateral frontal eye field (FEF), bilateral inferior parietal lobule (IPL), bilateral insula, right inferior frontal gyrus (IFG) and right temporoparietal junction (TPJ), suggesting attentional capture by the CS+ distractor (Table 1). Interestingly, structures of the basal ganglia previously linked to reward processing also preferentially responded to the CS+ distractor, including the bilateral substantia nigra (Figure 7A), bilateral nucleus accumbens (NAc), bilateral putamen and bilateral caudate tail (Figure 7B). In particular, the location of the caudate tail activations overlapped with the caudate tail regions implicated in value-driven attentional capture (Anderson et al., 2014). Signs of attentional bias were also evident when the CS+ distractor was present in the right hemifield (Table 2); in addition to the regions reported, the left lateral occipital cortex (Figure 7C) and right amygdala (Figure 7D) showed significant activations. These results suggest attentional bias generated by aversively-conditioned stimuli is associated with neural correlates very similar to those of value-driven attention.

A similar pattern emerged for the CS- distractor (Tables 3 and 4). The right TPJ, right IFG, bilateral anterior insula, bilateral substantia nigra, bilateral NAc, bilateral putamen and bilateral caudate tail responded preferentially when the CS- distractor was present in each hemifield. The amygdala showed significant activations only when the CS- distractor was present in the right hemifield. Since both the CS+ and CS- were passively presented in the training phase, this might be taken to suggest that the CS- was perceived as a safety signal and 


\section{MOTIVATIONAL SALIENCE GUIDES ATTENTION}

developed some degree of value-based attentional priority (Hackjin Kim, Shimojo, \& O'Doherty, 2006; Leknes, Lee, Berna, Andersson, \& Tracey, 2011; Navratilova \& Porreca, 2014).

Given their well-established role in threat (LeDoux, 1996; Vuilleumier, 2005) and reward processing (Schultz et al., 1997), respectively, we followed the significant activations in the amygdala and substantia nigra with ROI analyses. Bilateral amygdala and substantia nigra ROls were defined anatomically using the Talairach brain atlas (see Barbaro, Peelen, \& Hickey, 2017). Results confirmed that the two regions responded to both the CS+ and CS- distractors presented in each hemifield, all $t s>2.35$ and all $p s<0.05$.

Experiment 2. Experiment 1, in combination with previous research on value-driven attention (Anderson et al., 2011; Bucker \& Theeuwes, 2017; Haena Kim \& Anderson, 2019a; Le Pelley, Pearson, Griffiths, \& Beesley, 2015; Theeuwes \& Belopolsky, 2012), suggests that attentional capture by reward-related and aversively-conditioned stimuli recruit similar brain structures. Given the apparent regional overlap, we examined whether the two types of eliciting stimuli produce distinct patterns of activation using an MVPA in Experiment 2. We first contrasted the reward and shock distractors directly, but in no region did the response to a reward-and shock-associated distractor significantly differ. We then identified 11 ROls commonly activated by the reward and shock distractors (compared to a neutral distractor controlled for history as a former target), confirming the overlap suggested by Experiment 1. The 11 ROIs included the visual areas such as the extrastriate and primary visual cortex and the regions in the ventral and dorsal frontoparietal network including the TPJ, IFG, MFG, precuneus, FEF and intraparietal sulcus (IPS). Also included in the ROIs were the insula, caudate tail and thalamus (Figure 8 and Table 5). The MVPA revealed that the patterns of activation generated by the reward and shock distractors were not statistically distinguishable in any of the ROIs, all accuracy $<51.5 \%$, ps $>$ 0.18 (uncorrected for multiple comparisons).

A separate MVPA was performed using all of the clusters for which (a) reward distractors evoked stronger activation than neutral distractors and (b) shock distractors evoked stronger activation than neutral distractors (yellow+red and blue+red clusters in Figure 8) as ROls. If taskirrelevant reward and shock distractors are processed differently at all in the brain, then shock distractors should evoke a different pattern of activation in voxels significantly responsive to reward distractors than the reward distractors themselves and vice versa. This was not the case, however; classification accuracy was $49 \%, p=0.70$, for the reward $\mathrm{ROI}$ and $50 \%, p=0.47$, for the shock ROI ${ }^{1}$. A separate analysis demonstrated that the side on which the target was presented could be reliably classified in each of these two ROIs $(57.6 \%$ and $61.4 \%$, respectively, ps < 0.001), demonstrating that the lack of significant classification of reward vs. shock distractors was not due to a general insensitivity of the ROls ${ }^{2}$. A sensitivity power analysis (see Methods) indicated $80 \%$ power to detect a condition-specific increase in signal intensity (peak response) as small as $0.34 \%$ for the reward ROI and $0.38 \%$ for the shock ROI. Collectively, our neuroimaging results for Experiment 2 support the motivational relevance account that hypothesises that the

\footnotetext{
${ }^{1}$ Comparable results were obtained using an $\mathrm{ROI}$ defined by the conjoint activation of each of the three distractor conditions (reward, shock, neutral) against baseline (accuracy $=48.7 \%$ ). Comparable results were also obtained using regressors from a GLM in which the position of distractors was also modelled (i.e., separate regressors for when a given distractor appeared in the left and right hemifield) and MVPA was performed using ROls in only the contralateral hemisphere of the brain (averaging over left and right: accuracy $=51.5 \%, p=0.122$ for the reward ROls and $51.9 \%, p=0.087$ for the shock ROls [ $p$-values uncorrected for multiple comparisons]).

${ }^{2}$ Comparable results were obtained using a minimum statistic approach (Allefeld, Görgen, \& Haynes, 2016). Concerning target side, collapsing across the analyses using the two ROIs, we can reject the null hypothesis up to a prevalence of $\geq 0.435$. In contrast, concerning the valence of the distractors, we could not reject the null hypothesis for any prevalence $>0$ (this was also true of the analysis in which the position of the distractors was modelled).
} 


\section{MOTIVATIONAL SALIENCE GUIDES ATTENTION}

attentional system is primarily guided by motivational salience rather than a particular valence and thereby processes reward and threat cues similarly.

\section{Discussion}

The present study suggests that reward and aversive outcomes influence attention via a common mechanism, consistent with a motivational salience account of attentional control. By direct comparison, attention was biased toward stimuli previously associated with reward and threat to a comparable degree. Across two experiments, the presence of a task-irrelevant distractor previously associated with an aversive outcome activated the frontoparietal attentional network and the basal ganglia structures implicated in value-driven attentional capture. In Experiment 2, all of these regions exhibited similar patterns of activation in response to both reward and aversive distractors.

Separate lines of behavioural evidence concerning the influence of reward and aversive outcomes have suggested that they potentiate attentional bias in a similar manner. Stimuli previously associated with reward or aversive outcomes are attention riveting such that they disrupt performance even when they are unrelated to current task goals and not physically salient (Awh et al., 2012; Theeuwes, 2019). Their influence is not limited to attentional orienting (Anderson et al., 2011; Schmidt et al., 2015a; Theeuwes \& Belopolsky, 2012; Wentura et al., 2014) but also extends to action selection (Anderson, 2017a; Chapman, Gallivan, \& Enns, 2015; Haena Kim \& Anderson, 2019c) and is believed to emerge from value modulated activity within the visual cortex and basal ganglia (Anderson, 2017a, 2019). Outcome values associated with the stimuli induce plasticity within the visual cortex such that the stimuli are afforded priority in the saliency map (Anderson, 2017b, 2019; Anderson et al., 2014; Itthipuripat, Vo, Sprague, \& Serences, 2019; Pourtois, Schwartz, Seghier, Lazeyras, \& Vuilleumier, 2006); they also modulate the caudate tail activity which exerts control on oculomotor movement (Ghazizadeh, Griggs, \& Hikosaka, 2016; H. F. Kim \& Hikosaka, 2013; Yamamoto et al., 2013).

The present study complements previous findings by comparing the influence of reward and aversive outcomes simultaneously in a single paradigm and critically extends this work by directly comparing and contrasting the neural correlates. In Experiment 1, the CS+ distractor activated the brain regions in the frontoparietal attentional network including the anterior insula, TPJ, IFG, IPL and FEF, consistent with the dorsal/ventral attentional system view that activation in the dorsal frontoparietal regions along with the right TPJ, IFG and anterior insula reflects attentional orienting to a salient stimulus (Corbetta \& Shulman, 2002; Shulman et al., 2009). We also observed activations in the regions recruited by value-driven attention, including the caudate tail, NAc, amygdala and substantia nigra. Importantly, these regions revealed no differential activation to reward and aversive distractors in Experiment 2, suggesting that the attentional system is primarily guided by motivational salience rather than separate systems for positive and negative valence. This conclusion is in accordance with growing evidence for valenceindependent representations of appetitive and aversive information in the brain (Leknes \& Tracey, 2008; Lindquist, Satpute, Wager, Weber, \& Barrett, 2016; Seeley et al., 2007).

Associative learning, which underlies both value- and threat-driven attention (Bucker \& Theeuwes, 2017; Haena Kim \& Anderson, 2019a, in press; Le Pelley et al., 2015), involves establishing a stimulus-response association. The attentional orienting response to a stimulus signalling reward or aversive outcomes is reflexive and persists even in the absence of outcome delivery (Anderson et al., 2011; Schmidt et al., 2015a; Theeuwes \& Belopolsky, 2012). In the case of reward learning, stimulus-evoked caudate activity which in turn triggers attentional orienting is strengthened by reward signals from the substantia nigra. After learning, the rewardassociated stimulus becomes sufficient to generate an orienting response (Hikosaka et al., 2006). In particular, the caudate tail is implicated in the learning process, given its function in encoding stimulus representation and reflexive orienting based on stable value representation (H. F. Kim \& Hikosaka, 2013; Yamamoto et al., 2013; Yamamoto, Monosov, Yasuda, \& Hikosaka, 2012). We believe a similar process underlies threat-driven attention. In addition to value-coding dopamine neurons which are excited by reward and suppressed by aversive outcomes and facilitate valence-specific action, there are motivational salience-coding dopamine neurons that excite to both reward and aversive outcomes and specialise in orienting (Bromberg-Martin et al., 2010; 


\section{MOTIVATIONAL SALIENCE GUIDES ATTENTION}

Horvitz, 2000). These dopamine neurons in the substantia nigra transmit motivational salience signals in response to an aversively-conditioned stimulus (Bromberg-Martin et al., 2010; Ghazizadeh et al., 2016). The salience signals reinforce the stimulus-orienting association in the caudate tail, such that the threat predictive stimulus evokes automatic attentional orienting.

Although the amygdala has traditionally been regarded as a region for processing negatively-valenced emotion (LeDoux, 1996; Vuilleumier, 2005), evidence suggests its function extends to appetitive processes as well (Davis \& Whalen, 2001; Paton, Belova, Morrison, \& Salzman, 2006). This makes the amygdala an ideal candidate for encoding motivational salience (Metereau \& Dreher, 2013; Ousdal et al., 2008). In addition, although the amygdala has traditionally been thought to engage in spatially non-specific emotional processes, recent research demonstrates it is equally capable of tracking spatial information (Ousdal et al., 2014; Peck \& Salzman, 2014) and guiding oculomotor movement via its connections with the basal ganglia structures (Maeda, Inoue, Kunimatsu, Takada, \& Hikosaka, 2020). A similar role is assumed for the NAc. The NAc is a core structure implicated in encoding motivational salience (Horvitz, 2000; Navratilova \& Porreca, 2014) and mediating action selection via the direct or indirect pathway (Floresco, 2015; Wenzel, Rauscher, Cheer, \& Oleson, 2015). It is also recruited in attentional orienting towards valence-independent salience signals like surprise (Shulman et al., 2009; Zink, Pagnoni, Martin, Dhamala, \& Berns, 2003). Together, these findings imply a role of the amygdala and NAc in facilitating attentional orienting based on motivational salience.

Our results are in contrast with studies that report distinct responses to appetitive and aversive outcomes (Barbaro et al., 2017; Delgado et al., 2000; Tom, Fox, Trepel, \& Poldrack, 2007). These studies often manipulate financial incentives, whose neural and psychological effects may be different from those of thermal pain and electric shock. Thermal pain and electric shock are primary, positive punishers which have immediate consequences at the time of delivery. On the other hand, financial loss is a secondary, negative punisher that has a consequence only at a later time point. Additionally, under a typical experimental setting, participants expect a net gain even if they experience sporadic losses during a task, with the avoidance of losses potentially facilitating negative reinforcement. This points out a limitation of our own study - that we compared reinforcers that belong to different dimensions (primary punishment and secondary reward) - although the fact that we still see comparable neural activation is arguably all the more striking as a result. Future research should consider matching for reinforcer dimensions, for example, using primary taste as both reward and punishment stimuli. Another limitation of the study is that support for the motivational salience account comes in part from a null result. We note that using a different (i.e., non-linear) approach to pattern analysis could produce a different pattern of results.

In conclusion, the present study highlights the importance of motivational salience in experience-driven attentional control. Stimuli associated with reward and aversive outcomes have analogous effects on behaviour and recruit the same brain regions within the frontoparietal attentional network and basal ganglia. Within these regions, the patterns of activation evoked by stimuli of positive and negative valence are indistinguishable, indicative of a common neural mechanism primarily guided by motivational salience. In light of these findings, prior characterisations of attention as being distinctly value-driven (Anderson, 2016, 2019) or supporting threat monitoring (LeDoux, 1996; Vuilleumier, 2005) need to be revisited.

\section{Funding}

This study was supported by NARSAD Young Investigator Grant 26008 to BAA, NIH grant R01DA046410 to BAA, and a Texas A\&M University Program to Enhance Scholarly and Creative Activities (PESCA) grant to BAA and VAM. NN was supported by the NSF Graduate Research Fellowship Program.

\section{Conflict of Interest}

The authors declare no conflicting interests. 


\section{MOTIVATIONAL SALIENCE GUIDES ATTENTION}

\section{Author Contributions}

HK, NN, VAM and BAA conceived of the experiment. HK, HA, and NN programmed the experiment and led data collection efforts. HK, NN, VAM and BAA analysed the data. HK drafted the manuscript, which NN, HA, VAM and BAA edited. 
MOTIVATIONAL SALIENCE GUIDES ATTENTION

\section{References}

Allefeld, C., Görgen, K., \& Haynes, J.-D. (2016). Valid population inference for information-based imaging: From the second-level t-test to prevalence inference. Neurolmage, 141, 378392.

Anderson, B. A. (2016). The attention habit: how reward learning shapes attentional selection. Annals of the New York Academy of Sciences, 1369(1), 24-39.

Anderson, B. A. (2017a). Going for It: The Economics of Automaticity in Perception and Action. Current Directions in Psychological Science, 26(2), 140-145.

Anderson, B. A. (2017b). Reward processing in the value-driven attention network: reward signals tracking cue identity and location. Social Cognitive and Affective Neuroscience, 12(3), 461-467.

Anderson, B. A. (2019). Neurobiology of value-driven attention. Current Opinion in Psychology, 29, 27-33.

Anderson, B. A., Kuwabara, H., Wong, D. F., Gean, E. G., Rahmim, A., Brašić, J. R., et al. (2016). The Role of Dopamine in Value-Based Attentional Orienting. Current Biology, 26(4), 550-555.

Anderson, B. A., Kuwabara, H., Wong, D. F., Roberts, J., Rahmim, A., Brašić, J. R., et al. (2017). Linking dopaminergic reward signals to the development of attentional bias: A positron emission tomographic study. Neurolmage, 157, 27-33.

Anderson, B. A., Laurent, P. A., \& Yantis, S. (2011). Value-driven attentional capture. Proceedings of the National Academy of Sciences, 108(25), 10367-10371.

Anderson, B. A., Laurent, P. A., \& Yantis, S. (2014). Value-driven attentional priority signals in human basal ganglia and visual cortex. Brain Research, 1587, 88-96.

Awh, E., Belopolsky, A. V., \& Theeuwes, J. (2012). Top-down versus bottom-up attentional control: a failed theoretical dichotomy. Trends in Cognitive Sciences, 16(8), 437-443.

Baliki, M. N., Geha, P. Y., Fields, H. L., \& Apkarian, A. V. (2010). Predicting Value of Pain and Analgesia: Nucleus Accumbens Response to Noxious Stimuli Changes in the Presence of Chronic Pain. Neuron, 66(1), 149-160.

Barbaro, L., Peelen, M. V., \& Hickey, C. (2017). Valence, Not Utility, Underlies Reward-Driven Prioritization in Human Vision. The Journal of Neuroscience, 37(43), 10438.

Becerra, L., \& Borsook, D. (2008). Signal valence in the nucleus accumbens to pain onset and offset. European Journal of Pain, 12(7), 866-869.

Becerra, L., Breiter, H. C., Wise, R., Gonzalez, R. G., \& Borsook, D. (2001). Reward Circuitry Activation by Noxious Thermal Stimuli. Neuron, 32(5), 927-946.

Berridge, K. C., \& Robinson, T. E. (1998). What is the role of dopamine in reward: hedonic impact, reward learning, or incentive salience? Brain Research Reviews, 28(3), 309-369.

Bromberg-Martin, E. S., Matsumoto, M., \& Hikosaka, O. (2010). Dopamine in Motivational Control: Rewarding, Aversive, and Alerting. Neuron, 68(5), 815-834.

Brooks, J. C. W., Nurmikko, T. J., Bimson, W. E., Singh, K. D., \& Roberts, N. (2002). fMRI of Thermal Pain: Effects of Stimulus Laterality and Attention. Neurolmage, 15(2), 293-301.

Bucker, B., \& Theeuwes, J. (2017). Pavlovian reward learning underlies value driven attentional capture. Attention, Perception, \& Psychophysics, 79(2), 415-428.

Chapman, C. S., Gallivan, J. P., \& Enns, J. T. (2015). Separating value from selection frequency in rapid reaching biases to visual targets. Visual Cognition, 23(1-2), 249-271.

Chen, M., \& Bargh, J. A. (1999). Consequences of Automatic Evaluation: Immediate Behavioral Predispositions to Approach or Avoid the Stimulus. Personality and Social Psychology Bulletin, 25(2), 215-224.

Corbetta, M., \& Shulman, G. L. (2002). Control of goal-directed and stimulus-driven attention in the brain. Nature Reviews Neuroscience, 3, 201.

Davis, M., \& Whalen, P. J. (2001). The amygdala: vigilance and emotion. Molecular Psychiatry, $6(1), 13-34$.

Delgado, M. R., Nystrom, L. E., Fissell, C., Noll, D. C., \& Fiez, J. A. (2000). Tracking the Hemodynamic Responses to Reward and Punishment in the Striatum. 84(6), 3072-3077. 
Desimone, R., \& Duncan, J. (1995). Neural Mechanisms of Selective Visual Attention. Annual Review of Neuroscience, 18(1), 193-222.

Esterman, M., Tamber-Rosenau, B. J., Chiu, Y.-C., \& Yantis, S. (2010). Avoiding nonindependence in fMRI data analysis: Leave one subject out. Neurolmage, 50(2), 572576.

Floresco, S. B. (2015). The Nucleus Accumbens: An Interface Between Cognition, Emotion, and Action. 66(1), 25-52.

Freund, W., Klug, R., Weber, F., Stuber, G., Schmitz, B., \& Wunderlich, A. P. (2009). Perception and suppression of thermally induced pain: A fMRI study. Somatosensory \& Motor Research, 26(1), 1-10.

Gable, P., \& Harmon-Jones, E. (2010). The motivational dimensional model of affect: Implications for breadth of attention, memory, and cognitive categorisation. Cognition and Emotion, 24(2), 322-337.

Gardumi, A., Ivanov, D., Hausfeld, L., Valente, G., Formisano, E., \& Uludağ, K. (2016). The effect of spatial resolution on decoding accuracy in fMRI multivariate pattern analysis. Neurolmage, 132, 32-42.

Gaspelin, N., Leonard, C. J., \& Luck, S. J. (2015). Direct Evidence for Active Suppression of Salient-but-Irrelevant Sensory Inputs. Psychological Science, 26(11), 1740-1750.

Ghazizadeh, A., Griggs, W., \& Hikosaka, O. (2016). Ecological Origins of Object Salience: Reward, Uncertainty, Aversiveness, and Novelty. Frontiers in Neuroscience, 10(378).

Hikosaka, O., Nakamura, K., \& Nakahara, H. (2006). Basal Ganglia Orient Eyes to Reward. Journal of Neurophysiology, 95(2), 567-584.

Hikosaka, O., Takikawa, Y., \& Kawagoe, R. (2000). Role of the Basal Ganglia in the Control of Purposive Saccadic Eye Movements. Physiological Reviews, 80(3), 953-978.

Horvitz, J. C. (2000). Mesolimbocortical and nigrostriatal dopamine responses to salient nonreward events. Neuroscience, 96(4), 651-656.

Itthipuripat, S., Vo, V. A., Sprague, T. C., \& Serences, J. T. (2019). Value-driven attentional capture enhances distractor representations in early visual cortex. PLOS Biology, 17(8), e3000186.

Jensen, J., Mclntosh, A. R., Crawley, A. P., Mikulis, D. J., Remington, G., \& Kapur, S. (2003). Direct Activation of the Ventral Striatum in Anticipation of Aversive Stimuli. Neuron, 40(6), 1251-1257.

Jensen, K. B., Regenbogen, C., Ohse, M. C., Frasnelli, J., Freiherr, J., \& Lundström, J. N. (2016). Brain activations during pain: a neuroimaging meta-analysis of patients with pain and healthy controls. PAIN, 157(6).

Kim, H., \& Anderson, B. A. (2019a). Dissociable Components of Experience-Driven Attention. Current Biology, 29(5), 841-845.e842.

Kim, H., \& Anderson, B. A. (2019b). Dissociable neural mechanisms underlie value-driven and selection-driven attentional capture. Brain Research, 1708, 109-115.

Kim, H., \& Anderson, B. A. (2019c). Neural evidence for automatic value-modulated approach behaviour. Neurolmage, 189, 150-158.

Kim, H., \& Anderson, B. A. (in press). How does the attention system learn from aversive outcomes? Emotion.

Kim, H., Shimojo, S., \& O'Doherty, J. P. (2006). Is Avoiding an Aversive Outcome Rewarding? Neural Substrates of Avoidance Learning in the Human Brain. PLOS Biology, 4(8), e233.

Kim, H. F., \& Hikosaka, O. (2013). Distinct Basal Ganglia Circuits Controlling Behaviors Guided by Flexible and Stable Values. Neuron, 79(5), 1001-1010.

Le Pelley, M. E., Pearson, D., Griffiths, O., \& Beesley, T. (2015). When goals conflict with values: Counterproductive attentional and oculomotor capture by reward-related stimuli. Journal of Experimental Psychology: General, 144(1), 158-171.

LeDoux, J. E. (1996). The emotional brain: The mysterious underpinnings of emotional life. New York, NY, US: Simon \& Schuster.

Leknes, S., Lee, M., Berna, C., Andersson, J., \& Tracey, I. (2011). Relief as a Reward: Hedonic and Neural Responses to Safety from Pain. PLOS ONE, 6(4), e17870. 
Leknes, S., \& Tracey, I. (2008). A common neurobiology for pain and pleasure. Nature Reviews Neuroscience, 9(4), 314-320.

Lindquist, K. A., Satpute, A. B., Wager, T. D., Weber, J., \& Barrett, L. F. (2016). The Brain Basis of Positive and Negative Affect: Evidence from a Meta-Analysis of the Human Neuroimaging Literature. Cerebral Cortex, 26(5), 1910-1922.

Liu, X., Hairston, J., Schrier, M., \& Fan, J. (2011). Common and distinct networks underlying reward valence and processing stages: A meta-analysis of functional neuroimaging studies. Neuroscience \& Biobehavioral Reviews, 35(5), 1219-1236.

Maeda, K., Inoue, K.-i., Kunimatsu, J., Takada, M., \& Hikosaka, O. (2020). Primate AmygdaloNigral Pathway for Boosting Oculomotor Action in Motivating Situations. iScience, 23(6).

Mathur, V. A., Moayedi, M., Keaser, M. L., Khan, S. A., Hubbard, C. S., Goyal, M., et al. (2016). High Frequency Migraine Is Associated with Lower Acute Pain Sensitivity and Abnormal Insula Activity Related to Migraine Pain Intensity, Attack Frequency, and Pain Catastrophizing. 10(489).

Metereau, E., \& Dreher, J.-C. (2013). Cerebral Correlates of Salient Prediction Error for Different Rewards and Punishments. Cerebral Cortex, 23(2), 477-487.

Navratilova, E., \& Porreca, F. (2014). Reward and motivation in pain and pain relief. Nature Neuroscience, 17(10), 1304-1312.

Nissens, T., Failing, M., \& Theeuwes, J. (2017). People look at the object they fear: oculomotor capture by stimuli that signal threat. Cognition and Emotion, 31(8), 1707-1714.

O'Doherty, J. P. (2004). Reward representations and reward-related learning in the human brain: insights from neuroimaging. Current Opinion in Neurobiology, 14(6), 769-776.

Op de Beeck, H. P. (2010). Against hyperacuity in brain reading: Spatial smoothing does not hurt multivariate fMRI analyses? Neurolmage, 49(3), 1943-1948.

Ousdal, O. T., Jensen, J., Server, A., Hariri, A. R., Nakstad, P. H., \& Andreassen, O. A. (2008). The human amygdala is involved in general behavioral relevance detection: Evidence from an event-related functional magnetic resonance imaging Go-NoGo task. Neuroscience, 156(3), 450-455.

Ousdal, O. T., Specht, K., Server, A., Andreassen, O. A., Dolan, R. J., \& Jensen, J. (2014). The human amygdala encodes value and space during decision making. Neurolmage, 101, 712-719.

Paton, J. J., Belova, M. A., Morrison, S. E., \& Salzman, C. D. (2006). The primate amygdala represents the positive and negative value of visual stimuli during learning. Nature, 439(7078), 865-870.

Peck, C. J., \& Salzman, C. D. (2014). Amygdala neural activity reflects spatial attention towards stimuli promising reward or threatening punishment. eLife, 3, e04478.

Pourtois, G., Schwartz, S., Seghier, M. L., Lazeyras, F., \& Vuilleumier, P. (2006). Neural systems for orienting attention to the location of threat signals: An event-related fMRI study. Neurolmage, 31(2), 920-933.

Schmidt, L. J., Belopolsky, A. V., \& Theeuwes, J. (2015a). Attentional capture by signals of threat. Cognition and Emotion, 29(4), 687-694.

Schmidt, L. J., Belopolsky, A. V., \& Theeuwes, J. (2015b). Potential threat attracts attention and interferes with voluntary saccades. Emotion, 15(3), 329-338.

Schultz, W., Dayan, P., \& Montague, P. R. (1997). A Neural Substrate of Prediction and Reward. Science, 275(5306), 1593.

Seeley, W. W., Menon, V., Schatzberg, A. F., Keller, J., Glover, G. H., Kenna, H., et al. (2007). Dissociable Intrinsic Connectivity Networks for Salience Processing and Executive Control. The Journal of Neuroscience, 27(9), 2349.

Shulman, G. L., Astafiev, S. V., Franke, D., Pope, D. L. W., Snyder, A. Z., McAvoy, M. P., et al. (2009). Interaction of Stimulus-Driven Reorienting and Expectation in Ventral and Dorsal Frontoparietal and Basal Ganglia-Cortical Networks. The Journal of Neuroscience, 29(14), 4392.

Theeuwes, J. (2010). Top-down and bottom-up control of visual selection. Acta Psychologica, 135(2), 77-99. 


\section{MOTIVATIONAL SALIENCE GUIDES ATTENTION}

Theeuwes, J. (2019). Goal-driven, stimulus-driven, and history-driven selection. Current Opinion in Psychology, 29, 97-101.

Theeuwes, J., \& Belopolsky, A. V. (2012). Reward grabs the eye: Oculomotor capture by rewarding stimuli. Vision Research, 74, 80-85.

Tom, S. M., Fox, C. R., Trepel, C., \& Poldrack, R. A. (2007). The Neural Basis of Loss Aversion in Decision-Making Under Risk. Science, 315(5811), 515.

Vuilleumier, P. (2005). How brains beware: neural mechanisms of emotional attention. Trends in Cognitive Sciences, 9(12), 585-594.

Wager, T. D., Atlas, L. Y., Lindquist, M. A., Roy, M., Woo, C.-W., \& Kross, E. (2013). An fMRIBased Neurologic Signature of Physical Pain. 368(15), 1388-1397.

Wentura, D., Müller, P., \& Rothermund, K. (2014). Attentional capture by evaluative stimuli: Gainand loss-connoting colors boost the additional-singleton effect. Psychonomic Bulletin \& Review, 21(3), 701-707.

Wenzel, J. M., Rauscher, N. A., Cheer, J. F., \& Oleson, E. B. (2015). A Role for Phasic Dopamine Release within the Nucleus Accumbens in Encoding Aversion: A Review of the Neurochemical Literature. ACS Chemical Neuroscience, 6(1), 16-26.

Wolfe, J. M., Cave, K. R., \& Franzel, S. L. (1989). Guided search: An alternative to the feature integration model for visual search. Journal of Experimental Psychology: Human Perception and Performance, 15(3), 419-433.

Woo, C.-W., Schmidt, L., Krishnan, A., Jepma, M., Roy, M., Lindquist, M. A., et al. (2017). Quantifying cerebral contributions to pain beyond nociception. Nature Communications, 8(1), 14211.

Xu, K. Z., Anderson, B. A., Emeric, E. E., Sali, A. W., Stuphorn, V., Yantis, S., et al. (2017). Neural Basis of Cognitive Control over Movement Inhibition: Human fMRI and Primate Electrophysiology Evidence. Neuron, 96(6), 1447-1458.e1446.

Yacubian, J., Gläscher, J., Schroeder, K., Sommer, T., Braus, D. F., \& Büchel, C. (2006). Dissociable Systems for Gain- and Loss-Related Value Predictions and Errors of Prediction in the Human Brain. The Journal of Neuroscience, 26(37), 9530.

Yamamoto, S., Kim, H. F., \& Hikosaka, O. (2013). Reward Value-Contingent Changes of Visual Responses in the Primate Caudate Tail Associated with a Visuomotor Skill. The Journal of Neuroscience, 33(27), 11227.

Yamamoto, S., Monosov, I. E., Yasuda, M., \& Hikosaka, O. (2012). What and Where Information in the Caudate Tail Guides Saccades to Visual Objects. The Journal of Neuroscience, 32(32), 11005.

Zink, C. F., Pagnoni, G., Martin, M. E., Dhamala, M., \& Berns, G. S. (2003). Human Striatal Response to Salient Nonrewarding Stimuli. The Journal of Neuroscience, 23(22), 8092. 


\section{MOTIVATIONAL SALIENCE GUIDES ATTENTION}

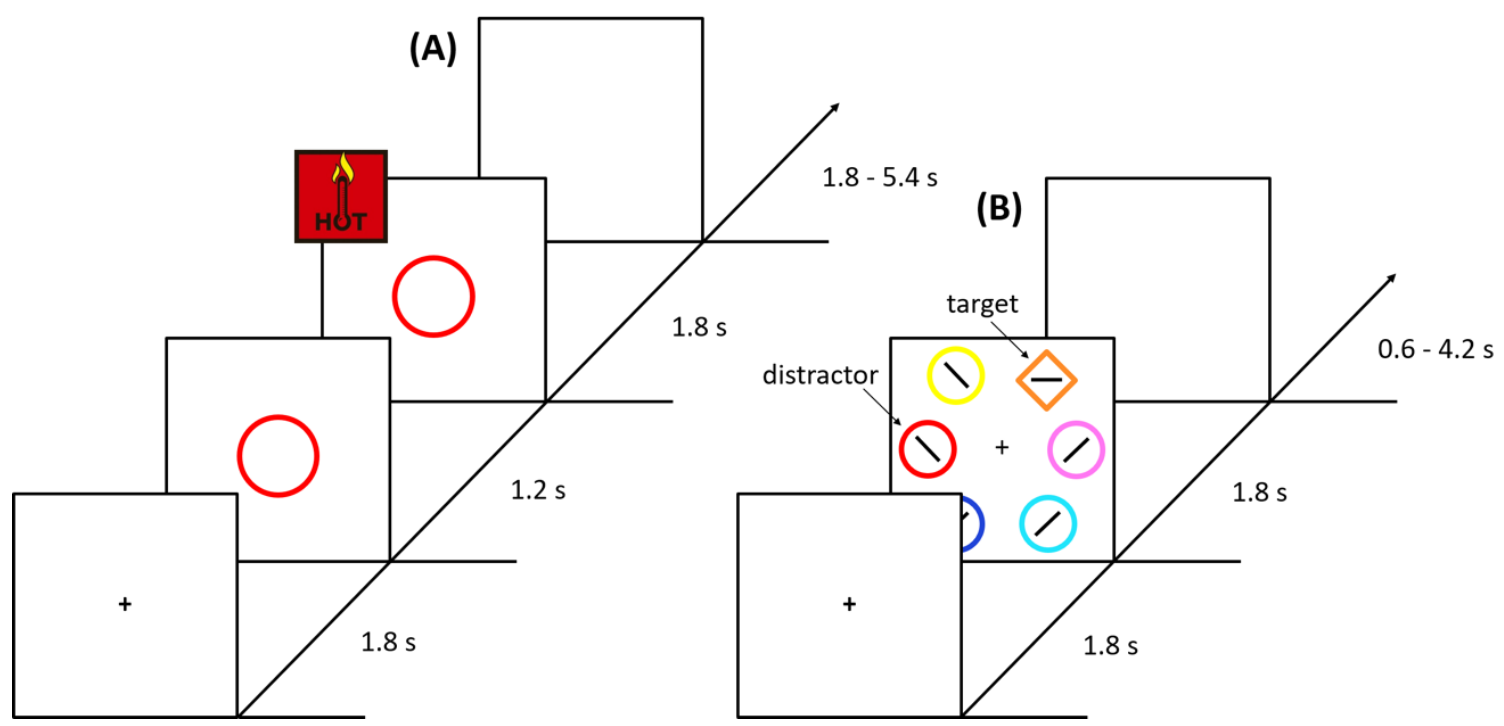

Figure 1. Sequence of events for a sample trial. (A) In the training phase, CS+ coloured circles were followed by a heat pulse that gradually increased for two seconds to reach the peak temperature, plateaued for two seconds then gradually decreased back to the baseline for two seconds. There were 30 trials in each run, half of which was CS+ trials. No heat stimulus was delivered on $1 / 3$ of the CS+ trials. (B) In the test phase, participants searched for a shape-defined target among non-salient distractors. There were 60 trials in each run. On 2/3 of the trials, one of the distractors appeared in either the CS+ colour or CS- colour (equally often). No CS distractor was present on the remaining trials. Participants completed five runs. 


\section{MOTIVATIONAL SALIENCE GUIDES ATTENTION}

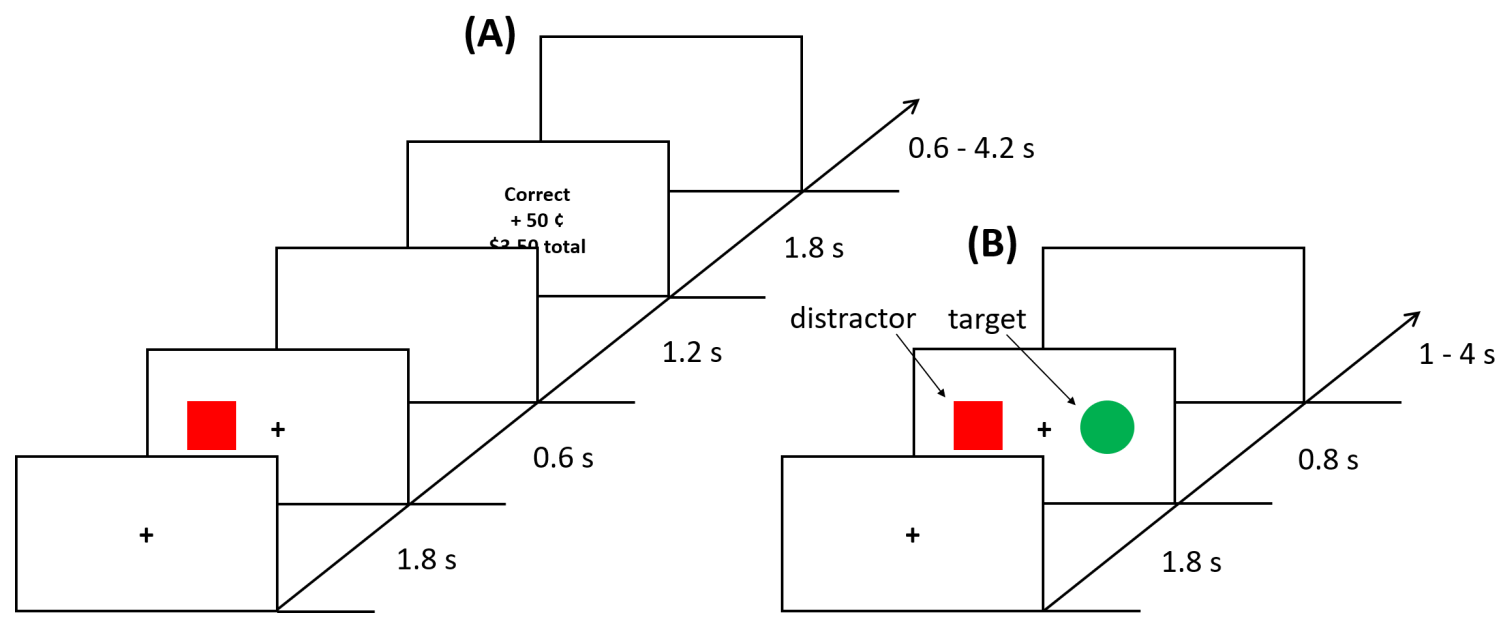

Figure 2. Sequence of events for a sample trial. (A) Each run of the training phase consisted of 40 trials. Participants generated a saccade to the target square. Feedback ("correct" or "incorrect") was provided on every trial. One colour was associated with reward, one with shock, and two with neither outcome (neutral). On $80 \%$ of reward-colour trials, monetary reward was delivered if participants responded correctly. On $80 \%$ of shock-colour trials, an electric shock was delivered simultaneously with the feedback. No monetary reward or shocks were ever delivered on neutral-colour trials. (B) Each run of the test phase consisted of 80 trials. On each trial, a square distractor and a circle target were presented simultaneously, one of which could appear in either the neutral, reward- or shock-associated colour, resulting in five target-distractor combinations (reward target-neutral distractor, shock target-neutral distractor, neutral targetneutral distractor, neutral target-reward distractor and neutral target-shock distractor).

Participants had to fixate the circle. A day prior to scanning, participants completed six runs of the training phase in the lab. During scanning, participants completed two training runs, three test runs, another training run and three test runs. 

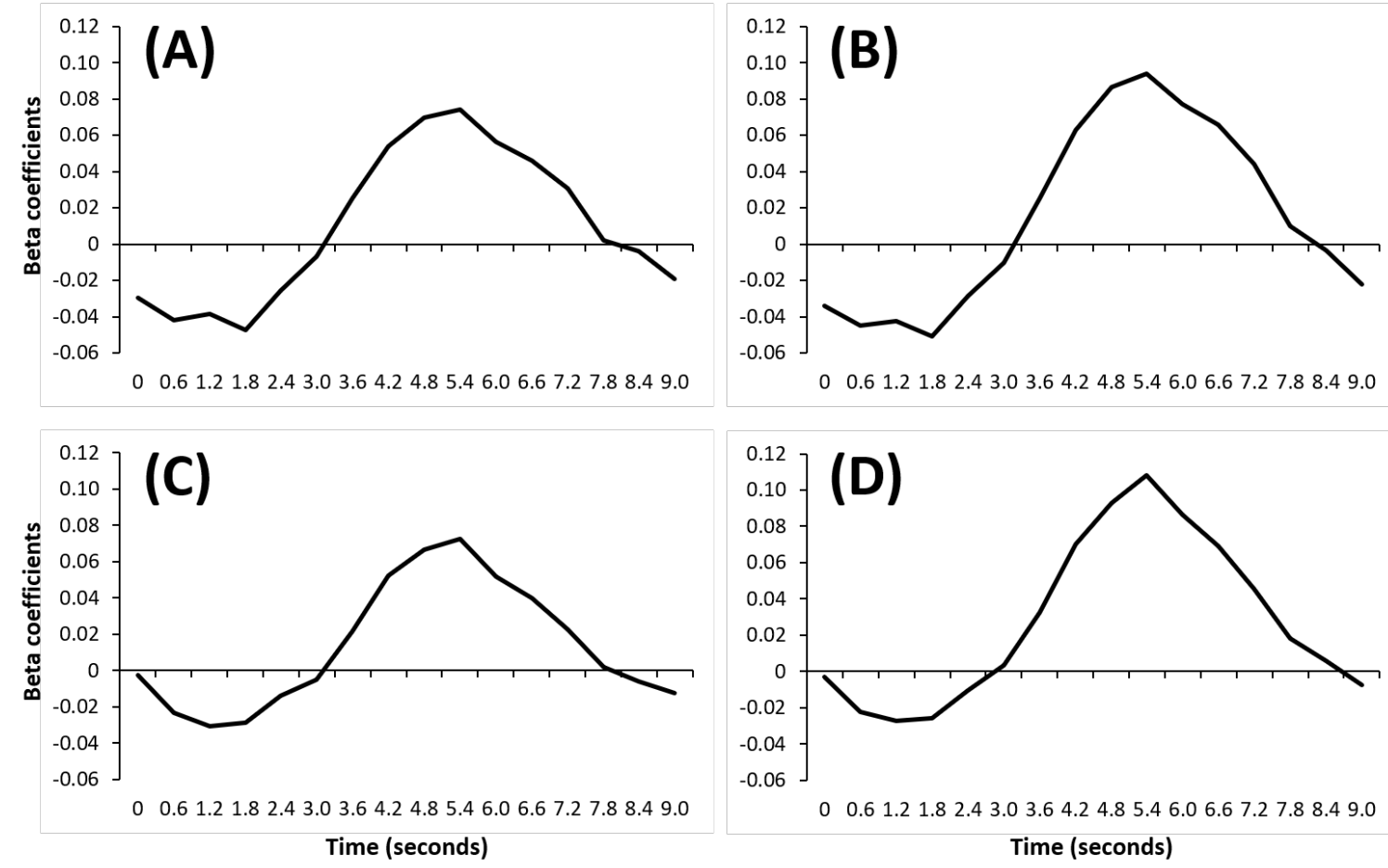

Figure 3. Top row: Average activation on reward distractor and neutral target trials in clusters for which $(A)$ reward distractors evoked stronger activation than neutral distractors and $(B)$ shock distractors evoked stronger activation than neutral distractors. Bottom row: Average activation on shock distractor and neutral target trials in clusters for which (C) reward distractors evoked stronger activation than neutral distractors and (D) shock distractors evoked stronger activation than neutral distractors. 
MOTIVATIONAL SALIENCE GUIDES ATTENTION

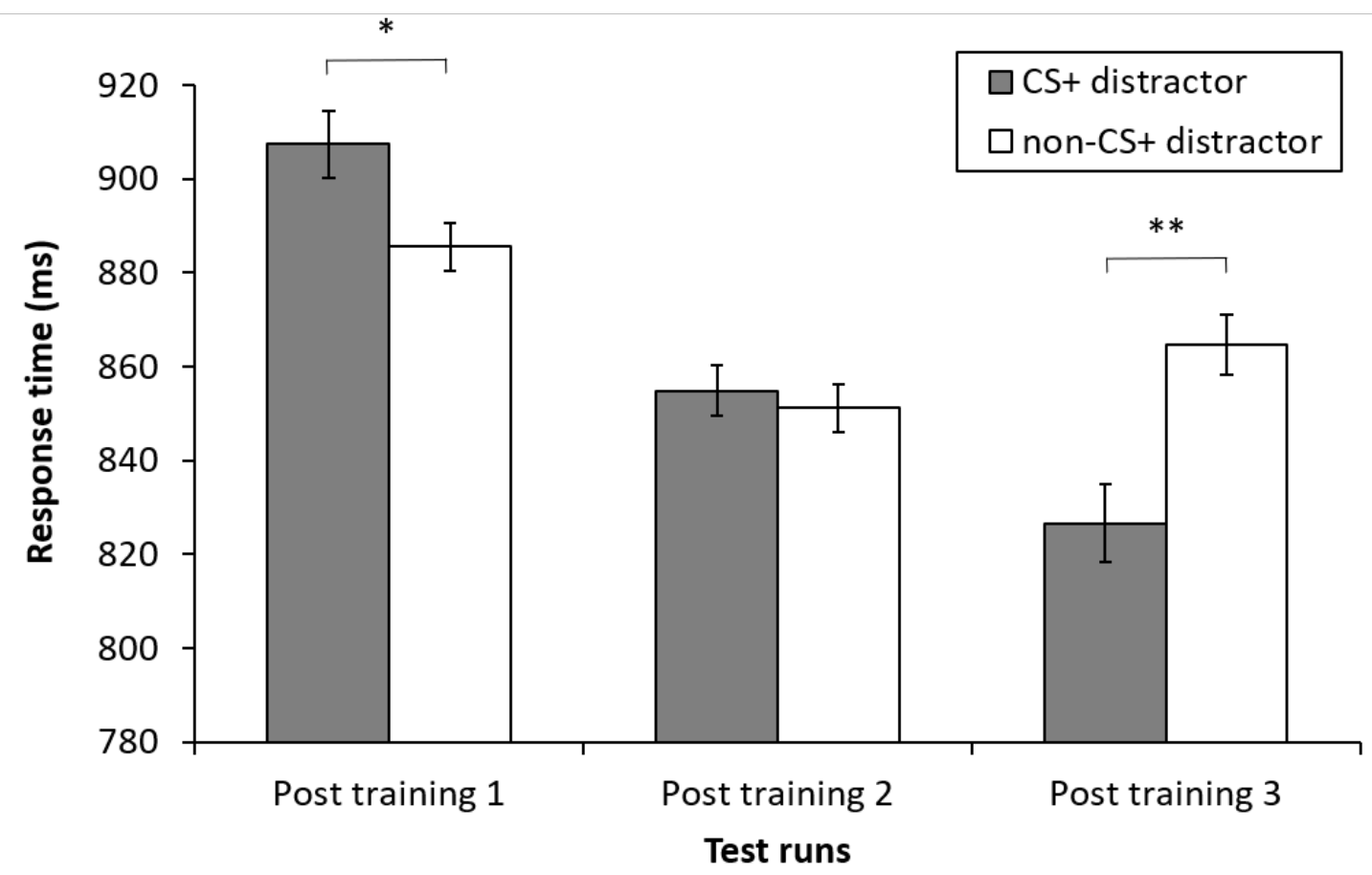

Figure 4. Mean response times in the test phase in Experiment 1. Error bars represent the withinsubjects SEM. ${ }^{*} p<0.01,{ }^{* *} p<0.001$ 


\section{MOTIVATIONAL SALIENCE GUIDES ATTENTION}

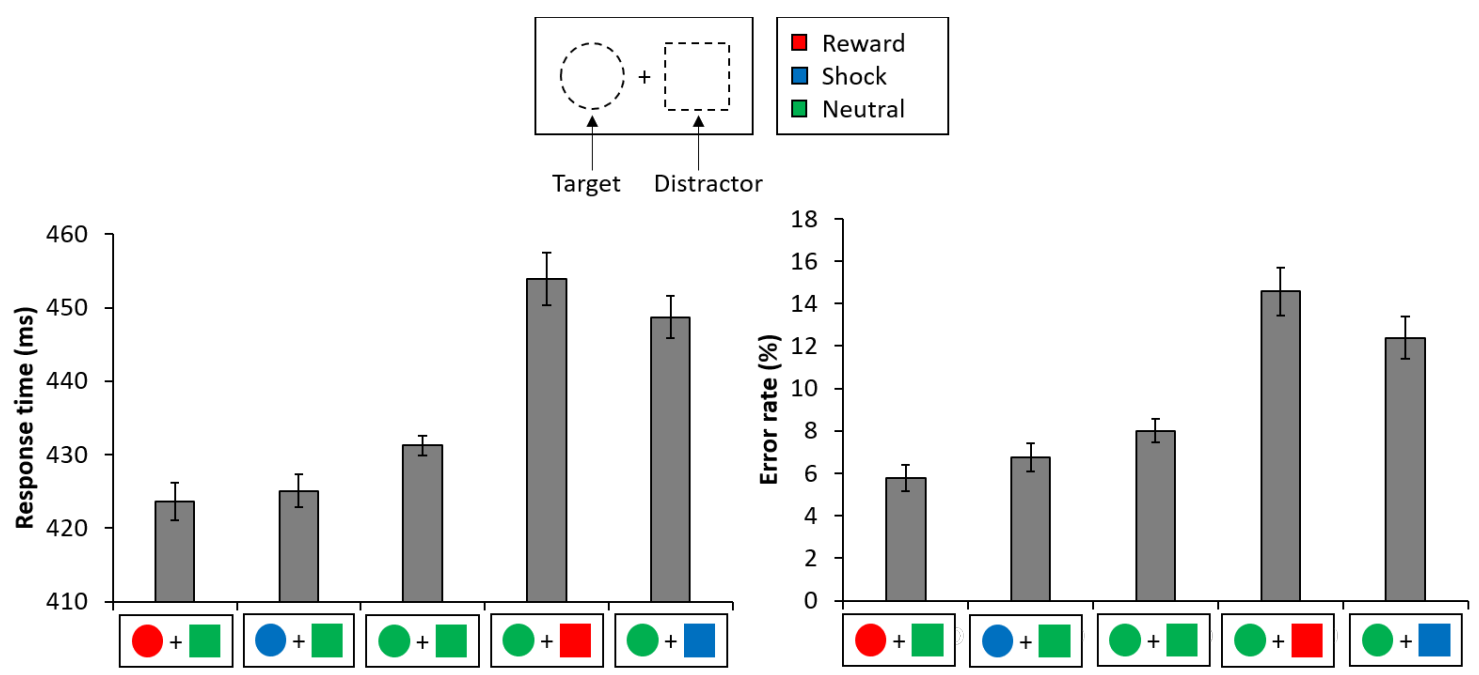

Figure 5. Mean response times (left) and error rates (right) in the test phase in Experiment 2. Error bars represent the within-subjects SEM. 

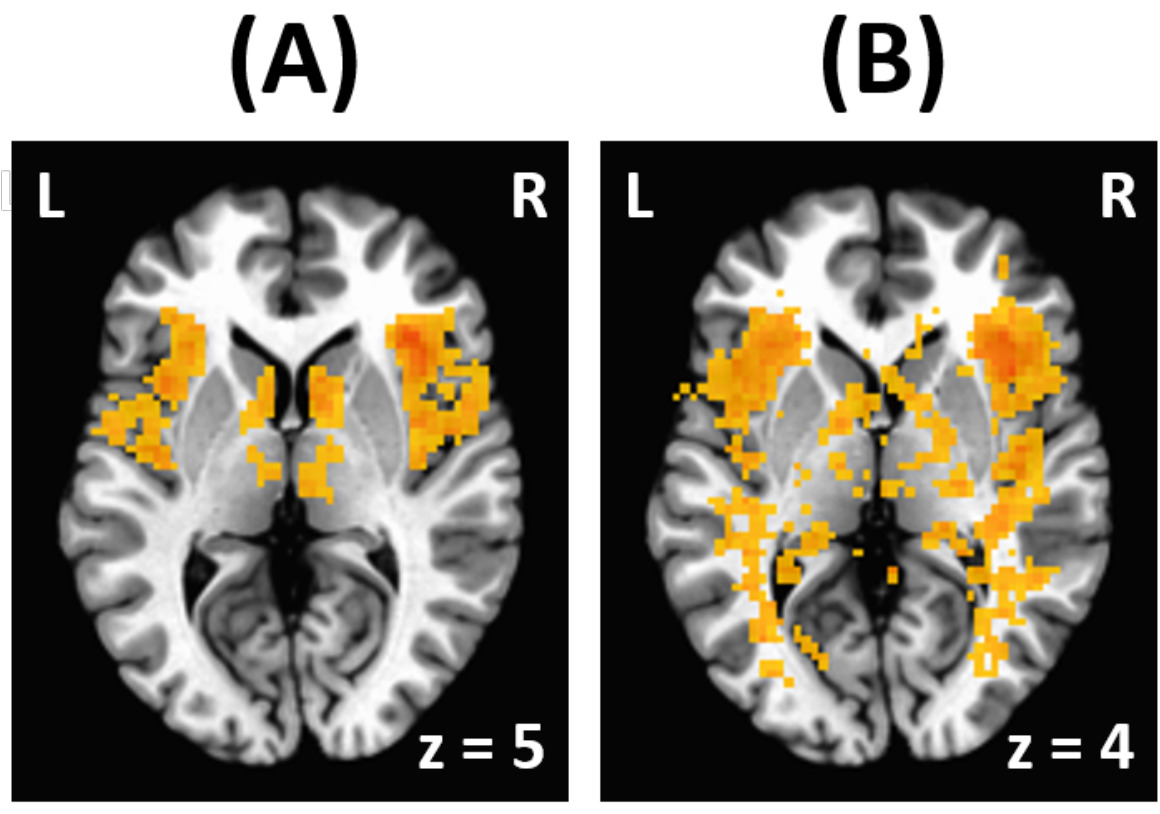

Figure 6. Brain regions showing greater activation to the $C S+$ circle than CS- circle during the training phase in Experiment 1. (A) CS+ circle followed by a heat pulse minus CS- circle contrast (B) CS+ circle not followed by a heat pulse minus CS- circle contrast. 
(A)

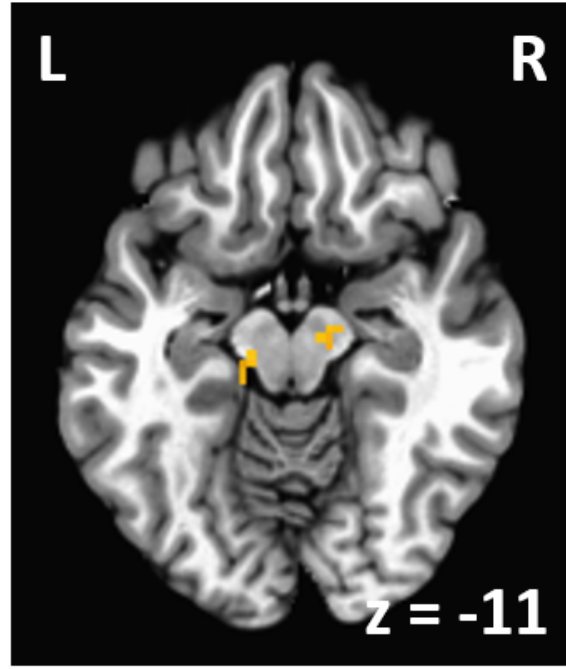

(C)

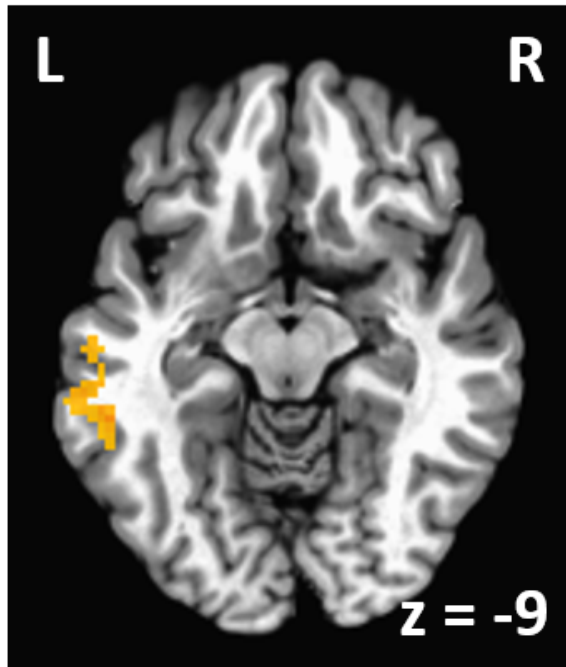

\section{(B)}

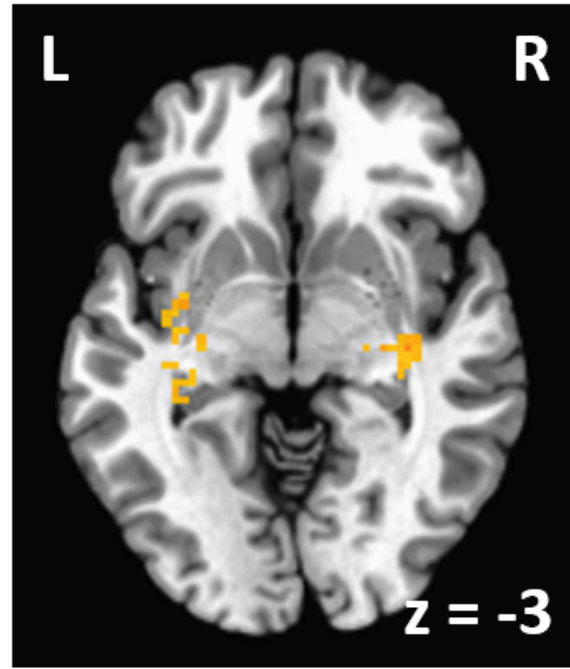

(D)

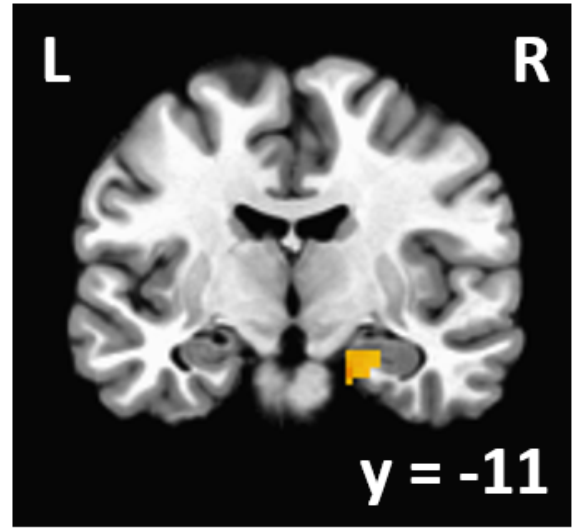

Figure 7. Brain regions showing greater activation when the $C S+$ distractor was present versus absent in the contralateral hemifield during the test phase in Experiment 1. (A) and (B) The CS+ distractor was present in the left hemifield. (C) and (D) The CS+ distractor was present in the right hemifield. 


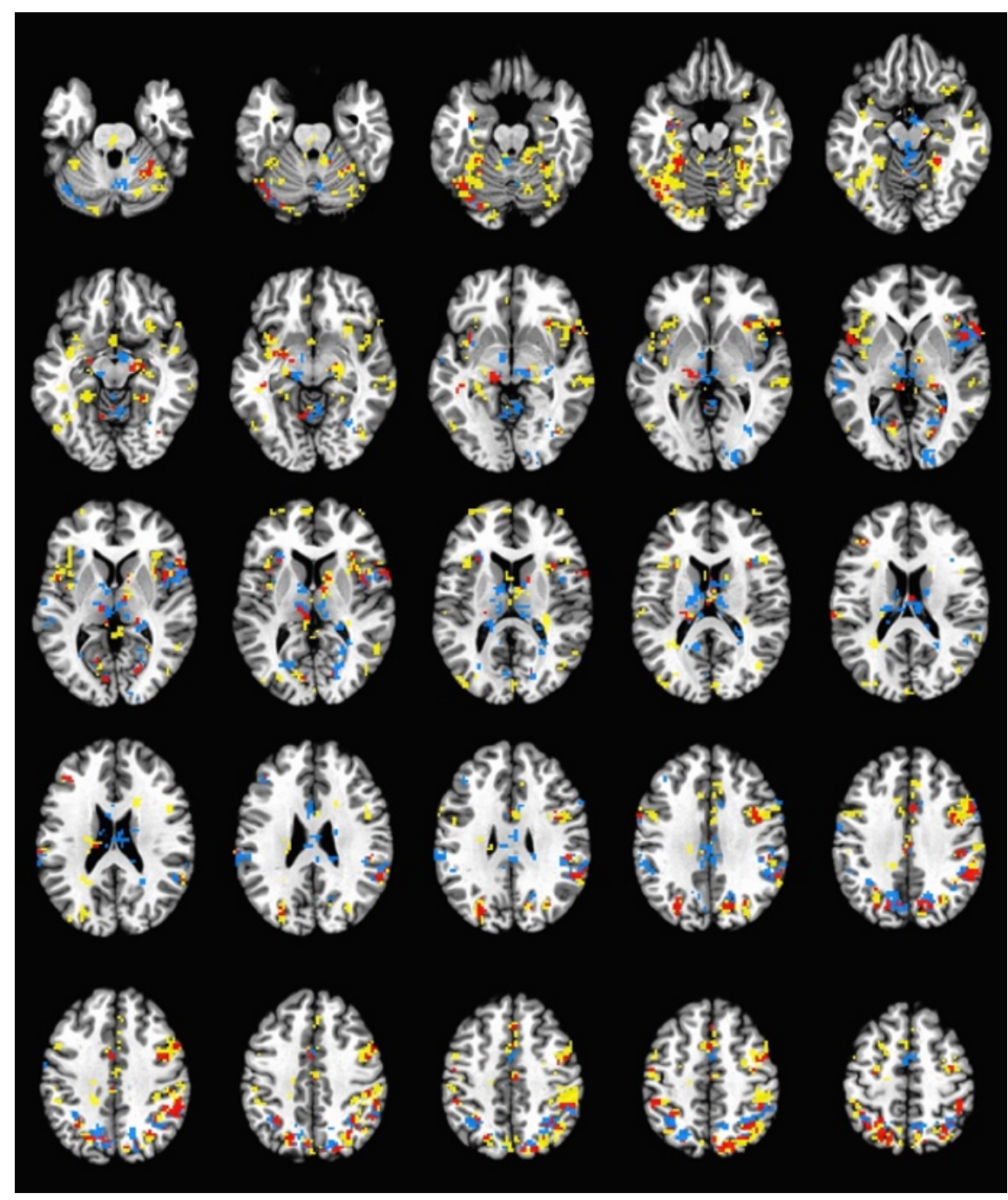

Figure 8. Distractor-evoked brain activation in Experiment 2. Yellow regions showed increased activation to the reward distractor and blue regions to the shock distractor compared to all neutral stimuli. Regions of overlap are shown in red. 


\section{MOTIVATIONAL SALIENCE GUIDES ATTENTION}

Table 1. Brain regions showing preferential activation to the CS+ distractor presented in the left visual field during the test phase in Experiment 1.

\begin{tabular}{|c|c|c|c|c|c|}
\hline \multirow{2}{*}{ Hemisphere } & \multirow{2}{*}{ Region } & \multicolumn{3}{|c|}{ Peak Talairach coordinates } & \multirow{2}{*}{ Volume $\left(\mathrm{mm}^{3}\right)$} \\
\hline & & $x$ & $y$ & $z$ & \\
\hline \multirow[t]{18}{*}{ Left } & anterior cingulate & -1 & 11 & -1 & 1781 \\
\hline & & -9 & 9 & 41 & 641 \\
\hline & & -16 & 31 & 24 & 391 \\
\hline & anterior insula & -29 & 9 & 6 & 453 \\
\hline & caudate tail & -31 & -19 & -6 & 1047 \\
\hline & & -31 & -26 & -1 & 672 \\
\hline & cerebellum & -4 & -41 & -31 & 734 \\
\hline & & -34 & -41 & -36 & 406 \\
\hline & frontal eye field & -19 & 1 & 54 & 375 \\
\hline & inferior frontal gyrus & -26 & 9 & 31 & 3531 \\
\hline & & -24 & 34 & -1 & 453 \\
\hline & middle occipital gyrus & -36 & -61 & -1 & 1906 \\
\hline & parahippocampal gyrus & -16 & -31 & -14 & 484 \\
\hline & postcentral gyrus & -29 & -21 & 34 & 5594 \\
\hline & posterior cingulate & -21 & -44 & 21 & 1422 \\
\hline & & -9 & -14 & 34 & 406 \\
\hline & superior parietal lobule & -19 & -41 & 59 & 453 \\
\hline & thalamus & -1 & -6 & -1 & 406 \\
\hline \multirow[t]{12}{*}{ Right } & anterior cingulate & 24 & 34 & 14 & 3297 \\
\hline & & 9 & -1 & 46 & 1563 \\
\hline & anterior insula & 31 & 9 & 14 & 3563 \\
\hline & caudate tail & 34 & -19 & -4 & 1359 \\
\hline & cerebellum & 11 & -46 & -29 & 391 \\
\hline & inferior frontal gyrus & 24 & 6 & 31 & 844 \\
\hline & & 39 & 39 & 6 & 531 \\
\hline & middle frontal gyrus & 26 & -1 & 46 & 391 \\
\hline & postcentral gyrus & 26 & -19 & 26 & 5047 \\
\hline & posterior cingulate & 14 & -36 & 21 & 984 \\
\hline & precuneus & 21 & -39 & 44 & 2953 \\
\hline & substantia nigra & 11 & -16 & -14 & 438 \\
\hline - & pons & 6 & -21 & -34 & 500 \\
\hline
\end{tabular}


MOTIVATIONAL SALIENCE GUIDES ATTENTION

Table 2. Brain regions showing preferential activation to the CS+ distractor presented in the right visual field during the test phase in Experiment 1.

\begin{tabular}{cccccc}
\hline \multirow{2}{*}{ Hemisphere } & \multirow{2}{*}{ Region } & \multicolumn{3}{c}{ Peak Talairach coordinates } & \multirow{2}{*}{ Volume $\left(\mathrm{mm}^{3}\right)$} \\
\cline { 3 - 5 } Left & anterior cingulate & -4 & $\mathrm{y}$ & $\mathrm{z}$ & 484 \\
& caudate body/tail & -16 & -19 & 24 & 1547 \\
& cerebellum & -9 & -69 & -29 & 703 \\
& & -39 & -66 & -21 & 500 \\
& & -14 & -24 & -36 & 391 \\
& middle frontal gyrus & -24 & 9 & 31 & 1000 \\
& middle occipital gyrus & -34 & -61 & -1 & 422 \\
& middle temporal gyrus & -51 & -39 & -9 & 1297 \\
& precuneus & -26 & -59 & 24 & 781 \\
& & -21 & -41 & 39 & 484 \\
& superior temporal gyrus & -36 & -49 & 16 & 1813 \\
& anterior cingulate & 16 & 29 & -1 & 375 \\
& caudate body & 16 & 4 & 24 & 1594 \\
& caudate tail & 24 & -29 & 16 & 516 \\
& cerebellum & 1 & -41 & -29 & 828 \\
& inferior frontal gyrus & 34 & 39 & 1 & 688 \\
& inferior parietal lobule & 34 & -41 & 29 & 3875 \\
& middle temporal gyrus & 31 & -64 & 11 & 438 \\
& parahippocampal gyrus & 16 & -14 & -21 & 750 \\
& posterior cingulate & 16 & -29 & 34 & 500 \\
& & 1 & -14 & 29 & 438 \\
\hline
\end{tabular}


Table 3. Brain regions showing preferential activation to the CS- distractor presented in the left visual field during the test phase in Experiment 1.

\begin{tabular}{|c|c|c|c|c|c|}
\hline \multirow{2}{*}{ Hemisphere } & \multirow{2}{*}{ Region } & \multicolumn{3}{|c|}{ Peak Talairach coordinates } & \multirow{2}{*}{ Volume $\left(\mathrm{mm}^{3}\right)$} \\
\hline & & $\mathrm{x}$ & $\mathrm{y}$ & $\mathrm{z}$ & \\
\hline \multirow[t]{17}{*}{ Left } & anterior cingulate & -4 & 34 & 9 & 484 \\
\hline & & -11 & 14 & 36 & 406 \\
\hline & caudate tail & -26 & -21 & 6 & 484 \\
\hline & cerebellum & -9 & -44 & -29 & 875 \\
\hline & & -6 & -56 & -39 & 438 \\
\hline & globus pallidus & -11 & 1 & -1 & 16125 \\
\hline & inferior frontal gyrus & -44 & 26 & 9 & 844 \\
\hline & & -39 & 14 & 19 & 719 \\
\hline & & -31 & 11 & 24 & 438 \\
\hline & inferior parietal lobule & -46 & -26 & 26 & 531 \\
\hline & medial frontal gyrus & -9 & 39 & 41 & 594 \\
\hline & posterior cingulate & -11 & -39 & 29 & 1063 \\
\hline & & -11 & -41 & 14 & 891 \\
\hline & precentral gyrus & -16 & -29 & 46 & 1031 \\
\hline & superior frontal gyrus & -6 & 29 & 51 & 422 \\
\hline & & -1 & 39 & 46 & 391 \\
\hline & superior temporal gyrus & -44 & -39 & 4 & 703 \\
\hline \multirow[t]{15}{*}{ Right } & anterior cingulate & 11 & 26 & -9 & 734 \\
\hline & & 19 & 34 & 11 & 563 \\
\hline & caudate tail & 26 & -11 & -4 & 828 \\
\hline & cerebellum & 9 & -41 & -1 & 391 \\
\hline & inferior frontal gyrus & 29 & 6 & 29 & 844 \\
\hline & & 44 & 34 & 1 & 594 \\
\hline & & 49 & 16 & 16 & 406 \\
\hline & medial frontal gyrus & 11 & 41 & 29 & 438 \\
\hline & middle frontal gyrus & 24 & 6 & 39 & 2547 \\
\hline & parahippocampal gyrus & 24 & -39 & 6 & 391 \\
\hline & posterior cingulate & 14 & -46 & 24 & 406 \\
\hline & precuneus & 14 & -39 & 54 & 1344 \\
\hline & substantia nigra & 11 & -16 & -11 & 891 \\
\hline & superior frontal gyrus & 14 & 24 & 59 & 1156 \\
\hline & superior temporal gyrus & 39 & -36 & 9 & 516 \\
\hline
\end{tabular}


Table 4. Brain regions showing preferential activation to the CS- distractor presented in the right visual field during the test phase in Experiment 1.

\begin{tabular}{|c|c|c|c|c|c|}
\hline \multirow{2}{*}{ Hemisphere } & \multirow{2}{*}{ Region } & \multicolumn{3}{|c|}{ Peak Talairach coordinates } & \multirow{2}{*}{ Volume $\left(\mathrm{mm}^{3}\right)$} \\
\hline & & $x$ & $\mathrm{y}$ & z & \\
\hline \multirow[t]{33}{*}{ Left } & amygdala & -21 & -6 & -16 & 609 \\
\hline & anterior cingulate & -9 & 29 & 4 & 2875 \\
\hline & & -14 & 36 & 21 & 641 \\
\hline & & -11 & 4 & 24 & 484 \\
\hline & anterior insula & -34 & 9 & -9 & 453 \\
\hline & caudate body & -14 & -9 & 26 & 1156 \\
\hline & & -21 & -6 & 24 & 453 \\
\hline & & -19 & 14 & 19 & 422 \\
\hline & caudate head & -6 & 6 & -1 & 1922 \\
\hline & cerebellum & -11 & -66 & -31 & 4734 \\
\hline & & -24 & -39 & -31 & 3750 \\
\hline & inferior frontal gyrus & -49 & 6 & 21 & 375 \\
\hline & inferior occipital gyrus & -24 & -91 & -9 & 1406 \\
\hline & inferior parietal lobule & -24 & -39 & 31 & 1281 \\
\hline & & -31 & -29 & 24 & 469 \\
\hline & lingual gyrus & -24 & -81 & -1 & 1813 \\
\hline & medial frontal gyrus & -14 & 14 & 44 & 438 \\
\hline & middle frontal gyrus & -41 & 19 & 26 & 1063 \\
\hline & middle occipital gyrus & -36 & -84 & 6 & 578 \\
\hline & & -26 & -79 & 16 & 469 \\
\hline & middle temporal gyrus & -54 & -36 & -11 & 1969 \\
\hline & paracentral lobule & -19 & -39 & 54 & 406 \\
\hline & parahippocampal gyrus & -26 & -36 & -14 & 6313 \\
\hline & posterior cingulate & -14 & -44 & 19 & 938 \\
\hline & & -9 & -6 & 34 & 563 \\
\hline & precentral gyrus & -46 & -4 & 34 & 781 \\
\hline & & -14 & -29 & 49 & 484 \\
\hline & precuneus & -24 & -61 & 21 & 3703 \\
\hline & superior temporal gyrus & -46 & 1 & -6 & 734 \\
\hline & & -41 & -16 & -9 & 594 \\
\hline & & -61 & -39 & 9 & 531 \\
\hline & & -39 & -4 & -11 & 484 \\
\hline & thalamus & -26 & -29 & 19 & 1125 \\
\hline \multirow[t]{12}{*}{ Right } & amygdala & 34 & -4 & -19 & 891 \\
\hline & & 26 & -14 & -16 & 531 \\
\hline & anterior cingulate & 14 & 26 & 26 & 469 \\
\hline & caudate head & 14 & 11 & 4 & 1484 \\
\hline & caudate tail & 34 & -16 & -11 & 672 \\
\hline & cerebellum & 14 & -34 & -26 & 2297 \\
\hline & & 1 & -36 & -29 & 1984 \\
\hline & & 14 & -39 & -49 & 953 \\
\hline & & 14 & -69 & -34 & 781 \\
\hline & & 26 & -61 & -34 & 766 \\
\hline & inferior frontal gyrus & 34 & 39 & 1 & 1734 \\
\hline & inferior parietal lobule & 36 & -36 & 26 & 2484 \\
\hline
\end{tabular}


MOTIVATIONAL SALIENCE GUIDES ATTENTION

\begin{tabular}{ccccc} 
lingual gyrus & 26 & -84 & 1 & 500 \\
medial frontal gyrus & 16 & -61 & -4 & 438 \\
middle frontal gyrus & 11 & 14 & 46 & 594 \\
& 31 & 1 & 36 & 4469 \\
middle occipital gyrus & 41 & 21 & 26 & 1328 \\
middle temporal gyrus & 29 & -84 & 16 & 422 \\
paracentral lobule & 46 & -39 & -1 & 6719 \\
postcentral gyrus & 4 & -34 & 51 & 797 \\
posterior cingulate & 24 & -29 & 44 & 422 \\
& 14 & -19 & 31 & 1484 \\
& 6 & -39 & 34 & 1422 \\
precentral gyrus & 19 & -21 & 39 & 484 \\
& 26 & 1 & 26 & 1688 \\
precuneus & 24 & -26 & 54 & 391 \\
& 24 & -59 & 36 & 6031 \\
putamen & 19 & -61 & 46 & 391 \\
puperior temporal gyrus & 24 & 9 & -4 & 406 \\
pons & 36 & 6 & -19 & 375 \\
& 9 & -14 & -31 & 1063 \\
\hline
\end{tabular}


MOTIVATIONAL SALIENCE GUIDES ATTENTION

Table 5. Regions of interest in Experiment 2.

\begin{tabular}{|c|c|c|c|c|c|}
\hline \multirow{2}{*}{ Hemisphere } & \multirow{2}{*}{ Region } & \multicolumn{3}{|c|}{ Centre of mass Talairach coordinates } & \multirow{2}{*}{ Volume $\left(\mathrm{mm}^{3}\right)$} \\
\hline & & $\mathrm{x}$ & $\mathrm{y}$ & z & \\
\hline \multirow[t]{25}{*}{ Left } & caudate tail & -30 & -5 & -6 & 47 \\
\hline & & -24 & -6 & -6 & 78 \\
\hline & extrastriate cortex & -41 & -69 & -16 & 78 \\
\hline & & -26 & -42 & -17 & 297 \\
\hline & & -25 & -71 & 28 & 500 \\
\hline & & -38 & -62 & -18 & 672 \\
\hline & frontal eye field & -28 & -14 & 56 & 484 \\
\hline & intraparietal sulcus & -38 & -46 & 50 & 63 \\
\hline & & -24 & -53 & 48 & 109 \\
\hline & & -44 & -38 & 45 & 281 \\
\hline & & -29 & -51 & 40 & 297 \\
\hline & & -34 & -55 & 49 & 297 \\
\hline & & -18 & -68 & 37 & 359 \\
\hline & & -16 & -63 & 50 & 891 \\
\hline & insula & -39 & -4 & 8 & 31 \\
\hline & & -35 & 11 & 9 & 31 \\
\hline & & -34 & 7 & -4 & 109 \\
\hline & & -46 & 5 & 3 & 297 \\
\hline & precuneus & -13 & -56 & 59 & 31 \\
\hline & & -6 & -69 & 36 & 219 \\
\hline & primary visual cortex & -8 & -77 & 7 & 94 \\
\hline & & -13 & -63 & 5 & 109 \\
\hline & thalamus & -19 & -25 & 14 & 188 \\
\hline & & -8 & -23 & 8 & 203 \\
\hline & & -14 & -23 & -2 & 422 \\
\hline \multirow[t]{21}{*}{ Right } & extrastriate cortex & 37 & -69 & -6 & 63 \\
\hline & & 25 & -42 & -13 & 344 \\
\hline & & 15 & -69 & 31 & 484 \\
\hline & frontal eye field & 37 & -10 & 48 & 156 \\
\hline & inferior frontal gyrus & 39 & 21 & -1 & 47 \\
\hline & & 41 & 25 & 2 & 47 \\
\hline & & 55 & 15 & 2 & 438 \\
\hline & intraparietal sulcus & 29 & -65 & 34 & 281 \\
\hline & & 32 & -59 & 44 & 1016 \\
\hline & insula & 31 & 18 & -2 & 109 \\
\hline & & 34 & 14 & 11 & 172 \\
\hline & & 44 & 7 & 5 & 500 \\
\hline & middle frontal gyrus & 40 & 1 & 35 & 891 \\
\hline & precuneus & 11 & -74 & 35 & 63 \\
\hline & & 8 & -60 & 55 & 94 \\
\hline & & 13 & -73 & 43 & 781 \\
\hline & primary visual cortex & 27 & -52 & 4 & 78 \\
\hline & & 19 & -70 & 7 & 172 \\
\hline & temporo-parietal junction & 50 & -43 & 36 & 1938 \\
\hline & thalamus & 8 & -13 & 6 & 63 \\
\hline & & 9 & -4 & 9 & 78 \\
\hline
\end{tabular}

